\title{
The effects of water and microstructure on the performance of polymer electrolyte fuel cells
}

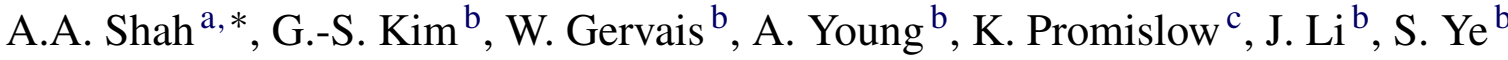 \\ a Department of Mathematics, Simon Fraser University, 8888 University Drive, BC, Canada V5A 1S6 \\ b Ballard Power Systems, 4343 North Fraser Way, Burnaby BC, Canada V5J $5 J 9$ \\ ${ }^{c}$ Department of Mathematics, Michigan State University, East Lansing, MI 48824, USA
}

Received 3 February 2006; received in revised form 21 February 2006; accepted 24 February 2006

Available online 18 April 2006

\begin{abstract}
In this paper, we present a comprehensive non-isothermal, one-dimensional model of the cathode side of a Polymer Electrolyte Fuel Cell. We explicitly include the catalyst layer, gas diffusion layer and the membrane. The catalyst layer and gas diffusion layer are characterized by several measurable microstructural parameters. We model all three phases of water, with a view to capturing the effect that each has on the performance of the cell. A comparison with experiment is presented, demonstrating excellent agreement, particularly with regard to the effects of water activity in the channels and how it impacts flooding and membrane hydration. We present several results pertaining to the effects of water on the current density (or cell voltage), demonstrating the role of micro-structure, liquid water removal from the channel, water activity, membrane and gas diffusion layer thickness and channel temperature. These results provide an indication of the changes that are required to achieve optimal performance through improved water management and MEA-component design. Moreover, with its level of detail, the model we develop forms an excellent basis for a multi-dimensional model of the entire membrane electrode assembly.
\end{abstract}

(C) 2006 Elsevier B.V. All rights reserved.

Keywords: PEMFC; Cathode catalyst layer; Water; Microstructure; Operating conditions; Performance prediction

\section{Introduction}

The fuel cell, in its various forms, is a promising alternative to the use of hydrocarbon fuels for energy production, motivated largely by concern for the environment. The proton exchange membrane fuel cell (PEMFC) receives a great deal of attention, particularly because it attains a relatively high power density and electrical efficiency. Before the benefits of this technology can be realized, several important practical issues need to be resolved, with the overarching aims of reducing the manufacturing cost and improving the performance and durability. A key concern, which we address in this paper, is the management of water in its various phases.

The heart of a single PEMFC is composed of a proton conducting membrane sandwiched between an anode and cathode. The anode and cathode are thin sheets of porous graphite paper, onto which a catalyst layer, composed roughly of carbon-grain

\footnotetext{
* Corresponding author. Tel.:+1 778288 4292; fax: +1 6042686657 .

E-mail address: ashah@pims.math.ca (A.A. Shah).
}

supported Platinum particles, is pressed. The basic principle of the PEMFC, or indeed any other fuel cell, is to convert chemical into electrical energy. At the anode, hydrogen is fed through the graphite paper, termed the gas diffusion layer (GDL), and is oxidized in the anode catalyst layer (ACL), producing hydrogen ions and free electrons. The electrons pass through an external circuit and the ions through the membrane, eventually combining with electrons and oxygen in the cathode catalyst layer (CCL) to form water. The oxygen is fed through a GDL at the cathode side. The membrane electrode assembly (MEA) is depicted in Fig. 1. Because of the low operating temperatures the Platinum $(\mathrm{Pt})$ catalyst is required to enhance the rate of reaction by providing an alternative pathway to the final products. The cost of Pt is clearly a concern; though the required amount of Pt has been reduced ten-fold reduction in the last few years, the percentage that is utilized is still very low (approximately $20 \%$ ).

The catalyst layers are amongst the most complex components of the fuel cell. Through the solid components (carbon grains, supporting smaller catalyst particles, and electrolyte), flow components of air and water vapor. Liquid water resides 


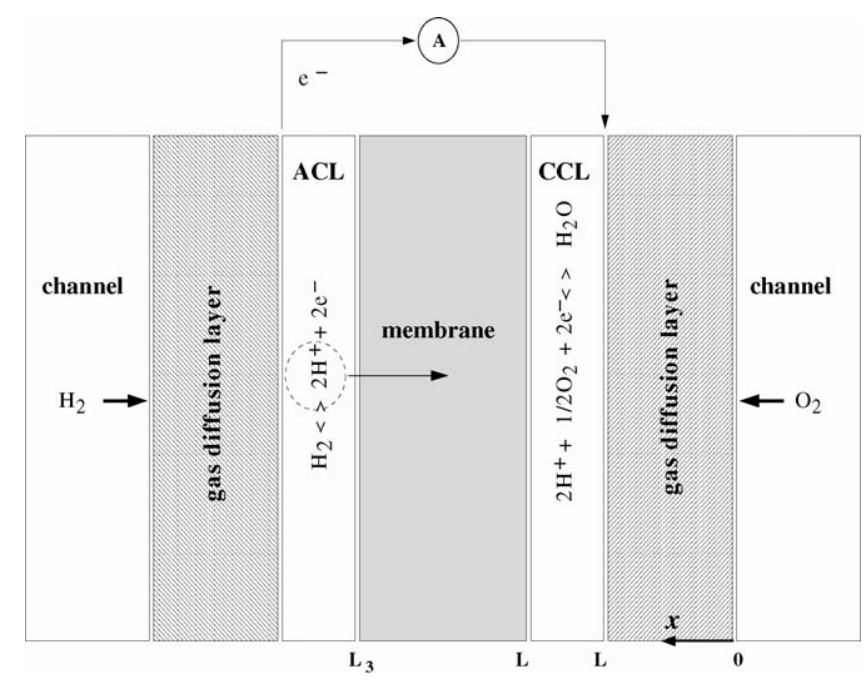

Fig. 1. Schematic representation of the MEA and a description of the geometry.

in the gas pores and dissolved water within the electrolyte. The electrons migrate through the carbon components of the layer while the protons migrate through pathways provided by the electrolyte. The latter is typically Nafion (a sulphonated Teflon), which contains both hydrophobic and hydrophilic regions, the second of which are vital in ensuring the free flow of the protons. The CCL is the more studied of the two catalyst layers for several reasons. The oxygen-reduction reaction (ORR) takes place at a much slower rate than the hydrogen oxidation at the anode and produces water. The hydrophilic regions in the CCL can cause water retention, which restricts the ingress of oxygen. Moreover, reaction is limited by the availability of $\mathrm{Pt}$, and can only occur at points of contact between the $\mathrm{Pt}$, carbon and electrolyte. Because of the importance of the CCL it is included explicitly in our model, with an attempt to capture some of the micro-scale features of its structure.

The properties of the membrane and the GDL also play a central role in the control of water. It is essential that the protons generated at the anode can reach the cathode freely, and in order to attain the high conductivity necessary to achieve this, the membrane must remain well hydrated. The GDL must allow sufficient oxygen from the cathode channel to enter the CCL, but must be sufficiently hydrophobic to expel any build-up of liquid water.

The preceding discussion underlines the unquestionable importance of water management, which in turn explains the growing emphasis placed on (particularly) liquid water in modelling studies, [1-9]. Though many of the issues are difficult to separate, they may be summarized as follows:

1. Flooding of the CCL and GDL and its impact on the performance;

2. The relationship between humidification of the cell and performance;

3. Hydration of the membrane and electrolyte (ionomer);

4. The trend of PEMFC operating conditions toward higher temperature and lower water activity, and their impact on saturation levels (optimal conditions for performance).
One of the difficulties associated with PEMFC modelling lies in accurately describing the structure of the CCL. The closest approximation to the structure is one of clumps of carbon grains (agglomerates) coated with and connected by electrolyte, $[6,10$ 14]. A widely employed approach in modelling the CCL is to assume that the agglomerates are spherical in shape, [6,7,15-20], and to incorporate the agglomerate-level activity into a homogeneous model by assuming that a thin film of electrolyte (or water) introduces a local resistance to the oxygen-motivated by the well-established theory of porous catalysts (see Chapter 3 of [21]). The three main variants are as follows:

- Include external resistance to the oxygen movement arising from the film(s) - this yields an expression for the limiting current density (or reaction rate) as a function of the agglomerate characteristics, $[3,6,7,15,18]$.

- Include internal resistance to the oxygen movement, due to flooding of the agglomerates with electrolyte or liquid water-this yields an effectiveness factor for the oxygen diffusion coefficient, [16,17].

- Include both internal and external resistances, as described above, [19,20].

Explicit incorporation of such resistances distinguishes these models from those in [22-28], which treat the CCL as a homogeneous porous medium, i.e., each phase exists in each reference control volume and is specified solely by a volume fraction. Such models are not able to capture the limiting-current-density phenomena.

A great deal of the recent modelling activity has been concentrated on multi-dimensional numerical simulation, noteworthy examples of which are $[2,4-7,9,18,19,27,29,30]$ (by no means an exhaustive list). The multi-dimensionality of these models significantly complicates their solution, forcing approximations to be made with respect to water and the CCL. Natarajan and Nguyen, [5], use a simplified and regularized form of the permeability in their isothermal model and do not explicitly model the membrane and CCL. He et al., [2], adopt a similar approach, simplifying further by assuming that the capillary diffusion coefficient is constant. Wang et al., [9], describe the water balance using the so-called multi-phase mixture $\left(\mathrm{M}^{2}\right)$ model, [31], where thermodynamic equilibrium between liquid water and vapour is assumed to prevail throughout. In other respects their model is similar to those in [5,2]. An isothermal $\mathrm{M}^{2}$ model is also used in [30], the focus of which is flooding in the GDL and CCL. Siegel et al., [6], incorporate the CCL and membrane, making the assumption that water exists only as vapour or as a species dissolved in the electrolyte/membrane, and are not able to account for liquid-water effects directly. The same is true of the model in [29], which treats all three forms of water as a single phase. In [7], Siegel et al. include all three forms but introduce several convoluted assumptions regarding the equilibrium between the three phases, as well as a highly regularized capillary diffusion coefficient. One-dimensional models that rigorously treat water can be found in the $[3,32,33]$. In the former, Lin et al. simplify the capillary diffusion coefficient, adopt isothermal conditions and assume a constant gas pressure. Nam and Kaviany, [32], 
concentrate on the GDL, treat the CCL as an interface and only introduce the membrane through the boundary conditions. They provide a thorough and rigorous discussion on the role of microstructural parameters in liquid-water movement. Pasaogullari et al., [33], employ the $\mathrm{M}^{2}$ model in a detailed study of water effects in the membrane and GDL, incorporating both anode and cathode. Their model is isothermal and treats the catalyst layer as an interface, focussing primarily on the effect of a micro-porous layer attached to the GDL.

In this paper we develop a comprehensive one-dimensional model, explicitly incorporating a GDL, CCL and membrane. Most of the features separately considered in detail in the models described above are included, as well as several new features, such as a separate equation for dissolved oxygen, nonequilibrium between water in the membrane and vapour, and membrane swelling. Our aim in this paper is to present the basic features of the model, and to demonstrate its success in capturing the convoluted water-driven phenomena. Extension of the model to two dimensions and to one of the entire MEA can be achieved in a straightforward manner.

The outline of this paper is as follows. In the next section we list the assumptions adopted in deriving the model and discuss the broader issues faced in MEA modelling. In Section 3 we present the model, with complete details and explanations. In Section 4 we describe the experimental procedure. The design of the cell is such that variations down the channel are minimal, i.e., pseudo one-dimensional, which allows comparison between modelling and experimental results to be made. The numerical results are then presented in Section 5, in which we first demonstrate agreement with the experiments. Finally, in Section 6 we provide a discussion and outline future work.

\section{Model description and assumptions}

Before embarking on the derivation of the model, it is instructive to list the factors that are commonly believed to impact MEA performance:

- the diffusion of oxygen through the pores of the GDL and CCL

- the transport of oxygen as a species dissolved in liquid water and electrolyte

- water production

- operating temperature and temperature variations

- the micro-structure of the GDL and CCL

- bulk convective motion of liquid water in response to pressure gradients

- water vapour in the channels and in the pores of the GDL and CCL

- hydration of the membrane

- electro-osmotic drag (migration)

- condensation/evaporation and absorption/desorption (to and from the electrolyte)

- the transport of protons and electrons

- flooding of the GDL and CCL pores

- membrane swelling
- non-uniform proton concentrations

- degradation issues, such as carbon oxidation and hydrogen peroxide production

- transient effects.

To simplify matters, we have chosen to neglect the last two items in this work. Their incorporation is the subject of current activity, in which the entire MEA is explicitly modelled. We say more on this in Section 6. We now describe the model and list the assumptions adopted in its derivation.

1. CCL characterization. As in the class of models discussed in the introduction, we assume that the carbon grains form spherical clusters (agglomerates) separated by primary pores. Surrounding the agglomerates is an electrolyte layer, on which a layer of liquid water can also exist. We assume that the contact between the carbon agglomerates is sufficient to ensure the free flow of protons and electrons, and that the connecting paths posses a negligible volume fraction. Within the agglomerates exists a fraction of the electrolyte. Highresolution electron microscopy confirms that the agglomerate structures are coated with electrolyte and some liquid water. Middelman, [34], postulates that the oxygen does not have access to the Pt particles located within the agglomerates, particularly those close to their centres. For reaction to occur within the agglomerates, sufficient electrolyte must be present, the quantity of which depends on the preparation process, [10,12,35], but is typically low (though [12,35] are two examples of efforts directed at increased electrolyte penetration into the agglomerates). We therefore assume that the $\mathrm{Pt}$ within the agglomerates is not active, i.e., that it does not contribute to the bulk reaction. It is possible to derive an effectiveness factor to remove this assumption. However, since it is unclear that significant reaction occurs within the agglomerates, and for the sake of simplicity, we continue with our assumption. If needed or justified, the inclusion of an effectiveness factor is straightforward.

2. Oxygen transport. Diffusion in large pores is assumed to be predominantly continuum, given their characteristic size and the typical operating pressures. Assuming that the oxy-

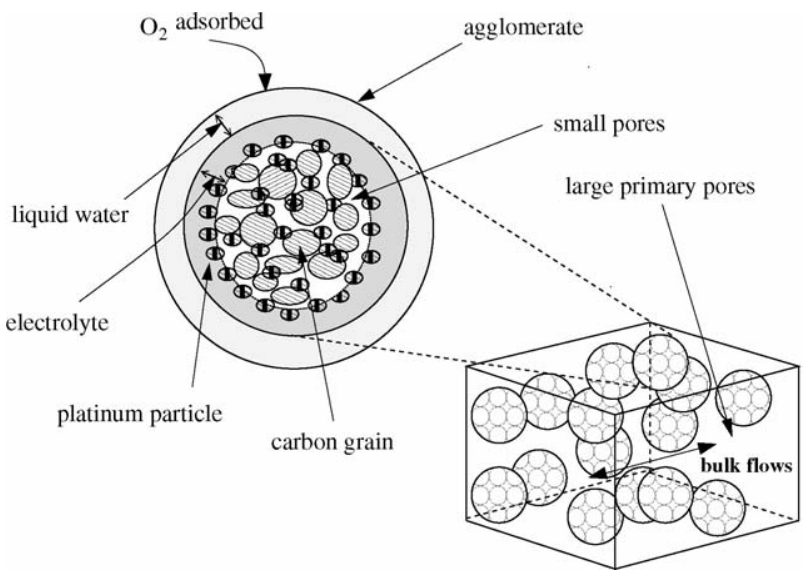

Fig. 2. The spherical-agglomerate structure assumed 
gen and water vapour are small concentrations in a nitrogen gas, we employ Fick's law. The oxygen in the primary pores reaches the Platinum particles on the agglomerate surface by dissolving in the water/electrolyte and diffusing across the layer(s). Henry's law is used to describe the equilibrium in oxygen concentration at the interface between the gas and liquid/solid phases (see Fig. 2).

3. Non-uniformity of proton concentration. The protons are transported in the form of hydronium ions, $\mathrm{H}_{3} \mathrm{O}^{+}$. We assume electro-neutrality (the number of negative charges equals the number of positive charges) but we do not make the typical assumption of a homogeneous distribution of charged sites in the electrolyte, which would allow us to take the concentration of $\mathrm{H}_{3} \mathrm{O}^{+}$as constant.

4. Water. The model accounts for water in all three forms; as a dissolved species, as vapour and as liquid. Liquid water resides in the primary pores. The water dissolved in the electrolyte is transported by diffusion (concentration gradients) and electro-osmotic drag (migration). As do Seigel et al., [6], we assume that the net water produced is either in liquid or vapour form. Condensation and evaporation are modelled using the approach in $[2,4,5]$, and references therein. In this representation the transfer occurs at a finite rate, dictated by the deviation of the local thermodynamic state from equilibrium. In a similar fashion, we introduce phase change between vapour and dissolved water by considering the deviation from equilibrium between dissolved water and vapour. Details for each are presented later.

5. Electrolyte volume. We include the effects of swelling on the volume of electrolyte in the CCL.

6. Temperature. The model is non-isothermal, which is necessary to model phase change accurately and to study the effects of water activity. We do however assume a single temperature for all phases.

Other assumptions are (a) negligibly small viscous flows, [36], and (b) a negligible ohmic potential drop in the electronically conducting carbon phase, justified by the relatively high carbon conductivity.

\section{Model derivation}

\subsection{Catalyst layer}

The geometry is depicted in Fig. 1, showing $x$ as the space variable, with the cathode channel placed at $x=0$, the GDL/CCL interface at $x=L_{1}$, the membrane/CCL interface at $x=L_{2}$ and the membrane/ACL interface at $x=L_{3}$.

Let $s$ be the saturation (fraction of primary pore volume occupied by liquid water), the concentrations of oxygen and nitrogen in the primary pores be $C_{\mathrm{p}}$ and $C_{\mathrm{N}}$, respectively, and the concentration of oxygen in the electrolyte be $C_{\mathrm{e}}$. Taking into account reaction, diffusion and absorption, mass balances yield:

$$
-\frac{\mathrm{d}}{\mathrm{d} x}\left((1-s) D_{\mathrm{p}} \frac{\mathrm{d} C_{\mathrm{p}}}{\mathrm{d} x}\right)=-\underbrace{h_{\mathrm{pe}}\left(H C_{\mathrm{p}}-C_{\mathrm{e}}\right)}_{\text {interfacial mass transfer }},
$$

$$
-\frac{\mathrm{d}}{\mathrm{d} x}\left(\epsilon_{\mathrm{e}} D_{\mathrm{e}} \frac{\mathrm{d} C_{\mathrm{e}}}{\mathrm{d} x}\right)=-\underbrace{\frac{1}{4} \mathcal{R}\left(\eta_{\mathrm{c}}, T, C_{\mathrm{e}}^{\mathrm{s}}\right)}_{\text {reactant consumption }}+h_{\mathrm{pe}}\left(H C_{\mathrm{p}}-C_{\mathrm{e}}\right),
$$

$-\frac{\mathrm{d}}{\mathrm{d} x}\left((1-s) D_{\mathrm{N}} \frac{\mathrm{d} C_{\mathrm{N}}}{\mathrm{d} x}\right)=0$,

where $\epsilon_{\mathrm{e}}$ is the volume fraction of electrolyte and $D_{\mathrm{p}}, D_{\mathrm{N}}$ and $D_{\mathrm{e}}$ are the molecular diffusion coefficients of oxygen and nitrogen through the primary pores and of oxygen through the electrolyte. The first are subject to a Bruggeman correction (in the absence of a value for the tortuosity of the flow path) and their dependence on temperature and pressure follows the Chapman-Enskog formula (in $\mathrm{m}^{2} \mathrm{~s}^{-1}$ ), [37],

$D_{\mathrm{p}}=3.8 \times 10^{-9} \frac{T^{3 / 2}}{P} \epsilon_{\mathrm{p}}^{3 / 2}, \quad D_{\mathrm{N}}=3.6 \times 10^{-9} \frac{T^{3 / 2}}{P} \epsilon_{\mathrm{p}}^{3 / 2}$,

where $\epsilon_{\mathrm{p}}$ is the volume fraction of primary pores, $T$ is temperature and $P$ is the gas pressure. The variations of $D_{\mathrm{p}}$ and $D_{\mathrm{N}}$ with temperature and pressure are neglected, that is, we use a constant value based on the temperature at the cathode channel. Since temperature and pressure variations are weak, the correspondingly small changes in $D_{\mathrm{p}}$ and $D_{\mathrm{N}}$ have a negligible effect. The coefficient $D_{\mathrm{e}}$ has the following form (taken form [38]):

$D_{\mathrm{e}}=3.1 \times 10^{-7} \mathrm{e}^{-2768 / T}$,

where once again we approximate temperature in this expression by its value at the cathode channel.

$\mathcal{R}\left(\eta_{\mathrm{c}}, T, C_{\mathrm{e}}^{\mathrm{s}}\right)$ is the reaction rate, based on the Butler-Volmer law, $h_{\text {pe }}$ is a volumetric mass transfer coefficient from gas to electrolyte (on the air side), and $H$ is a dimensionless Henry constant. The diffusive flux of oxygen from the pore space to the interface between the gas and electrolyte/water is balanced by the amount adsorbed into the electrolyte/water.

To prescribe the mass-transfer coefficient, $h_{\text {pe }}$, we use the formula for the Sherwood number given in [37] for flow past a spherical particle.

$S h=2+0.6 R e^{1 / 3} S c^{2 / 3}$,

where $R e$ is the Reynolds number and $S c$ is the Schmidt number. For a predominantly diffusive process, we can approximate $S h=2$. From the formula $h_{\mathrm{pe}} S_{\mathrm{a}}=S h D_{\mathrm{p}} / D_{\mathrm{ag}}$, where $D_{\mathrm{ag}}$ is the agglomerate diameter and $s_{\mathrm{a}}$ is the specific surface area of the agglomerates, we obtain $h_{\mathrm{pe}}=O\left(10^{5}\right)$. It should be noted that estimating mass transfer coefficients is itself a great challenge, a detailed discussion of which is beyond the scope of the present paper.

The electrolyte volume fraction, $\epsilon_{\mathrm{e}}$, will increase as it swells with water. We separate the electrolyte film that coats the agglomerates (volume fraction $\epsilon_{\mathrm{e}}^{\mathrm{f}}$ ) from that contained in the interiors (volume fraction $\epsilon_{\mathrm{e}}^{\mathrm{i}}$ ) and write $\epsilon_{\mathrm{e}}^{0}=\epsilon_{\mathrm{e}}^{\mathrm{f}}+\epsilon_{\mathrm{e}}^{\mathrm{i}}$. The Pt inside the agglomerates is assumed to be inactive, that is, there is a negligible surface area of $\mathrm{Pt}$ in contact with the fraction $\epsilon_{\mathrm{e}}^{\mathrm{i}}$ of electrolyte. The combined volume fraction of the carbon, Pt and small pores, $\epsilon_{\mathrm{a}}$, is assumed constant. The volume fraction of primary pores is then given by $\epsilon_{\mathrm{p}}=1-\epsilon_{\mathrm{a}}-\epsilon_{\mathrm{e}}$. The swelling of 
the electrolyte is assumed to impact on the thickness of the electrolyte film. To incorporate it we use the following approximate relationship:

$\epsilon_{\mathrm{e}}=\epsilon_{\mathrm{e}}^{0}+k_{\mathrm{s}} \lambda$,

where $k_{\mathrm{s}}=0.0126$, and $\lambda$ is the membrane water content ( $\mathrm{mol}$ $\mathrm{H}_{2} \mathrm{O} / \mathrm{mol} \mathrm{SO}_{3}^{-}$), given by

$\lambda=\frac{C_{\mathrm{d}}}{1-k_{\mathrm{s}} C_{\mathrm{d}}}$,

where $C_{\mathrm{d}}$ is the dissolved water concentration in the electrolyte, normalized with respect to $v=1800 \mathrm{~mol} \mathrm{~m}^{-3}$, the fixed-charge site concentration of the membrane, [39].

To derive an equation for the electrolyte potential in the CCL, $\phi$, we consider a mass balance for $\mathrm{H}_{3} \mathrm{O}^{+}$. With the assumption of a negligible convective flux, the contribution to the movement comes from migration and concentration gradients:

$-\frac{\mathrm{d}}{\mathrm{d} x} \underbrace{\left(\frac{\epsilon_{\mathrm{e}} \sigma_{\mathrm{e}}}{F} \frac{\mathrm{d} \phi}{\mathrm{d} x}+\epsilon_{\mathrm{e}} D_{\mathrm{H}} C_{\mathrm{d}} \frac{\mathrm{d} C_{\mathrm{H}}}{\mathrm{d} x}\right)}_{\text {diffusive flux of } \mathrm{H}_{3} \mathrm{O}^{+}}=-\underbrace{\mathcal{R}\left(\eta_{\mathrm{c}}, T, C_{\mathrm{e}}^{\mathrm{s}}\right)}_{\mathrm{H}_{3} \mathrm{O}^{+} \text {consumption }}$,

where $C_{\mathrm{H}}$ is the $\mathrm{H}_{3} \mathrm{O}^{+}$concentration, $\sigma_{\mathrm{e}}$ is the protonic conductivity of the electrolyte, $D_{\mathrm{H}}$ is the $\mathrm{H}_{3} \mathrm{O}^{+}$diffusion coefficient and $F$ is Faraday's constant. $C_{\mathrm{H}}$ and $C_{\mathrm{d}}$ are related as follows [40]

$C_{\mathrm{H}}=-\frac{1}{2} k_{\mathrm{e}} C_{\mathrm{d}}+\sqrt{\left(\frac{1}{2} k_{\mathrm{e}} C_{\mathrm{d}}\right)^{2}+k_{\mathrm{e}} C_{\mathrm{d}}}$,

where the temperature dependent $k_{\mathrm{e}}$ is given by

$k_{\mathrm{e}}=k_{0} \exp \left(\frac{H_{0}}{R}\left(\frac{1}{T}-\frac{1}{298}\right)\right)$.

As an approximation we assume that $k_{\mathrm{e}}$ is constant. Note that Eq. (9) is derived with the assumption of electro-neutrality. We refer to [41] for details. The diffusion coefficient $D_{\mathrm{H}}$ is taken from [40]:

$D_{\mathrm{H}}=1.6 \times 10^{-8} \mathrm{e}^{-1683 / T} C_{\mathrm{d}}$,

where once again we neglect the dependence on temperature variations. For $\sigma_{\mathrm{e}}$ we use the law derived in [39],

$\sigma_{\mathrm{e}}=\exp \left(1268\left(\frac{1}{303}-\frac{1}{T}\right)\right)(5.14 \lambda-0.326)$.

The algebraic term on the right-hand side of (12) accounts for the effect of hydration on the conductivity. It is based on empirical data collected by Springer et al. [39].

An equation for the temperature, $T$, is derived from an energy balance of conduction, convective heat flux from liquid water movement and heat sources

$$
\begin{aligned}
& -\frac{\mathrm{d}}{\mathrm{d} x}\left(k \frac{\mathrm{d} T}{\mathrm{~d} x}-s \epsilon_{\mathrm{p}} \rho_{\mathrm{l}} C_{1} v_{\mathrm{l}} T\right)=\underbrace{\left(\frac{(-\delta s) T}{n_{\mathrm{e}}}+F \eta\right) \mathcal{R}\left(\eta_{\mathrm{c}}, T, C_{\mathrm{e}}^{\mathrm{s}}\right)}_{\text {reaction and activation loss }} \\
& +\underbrace{\epsilon_{\mathrm{e}} \sigma_{\mathrm{e}}\left(\frac{\mathrm{d} \phi}{\mathrm{d} x}\right)^{2}}_{\text {ohmic heating }}+\underbrace{h_{\mathrm{gl}} h_{\mathrm{pc}}\left(R T C_{\mathrm{v}}-P_{\mathrm{sat}}\right)}_{\text {phase change }} .
\end{aligned}
$$

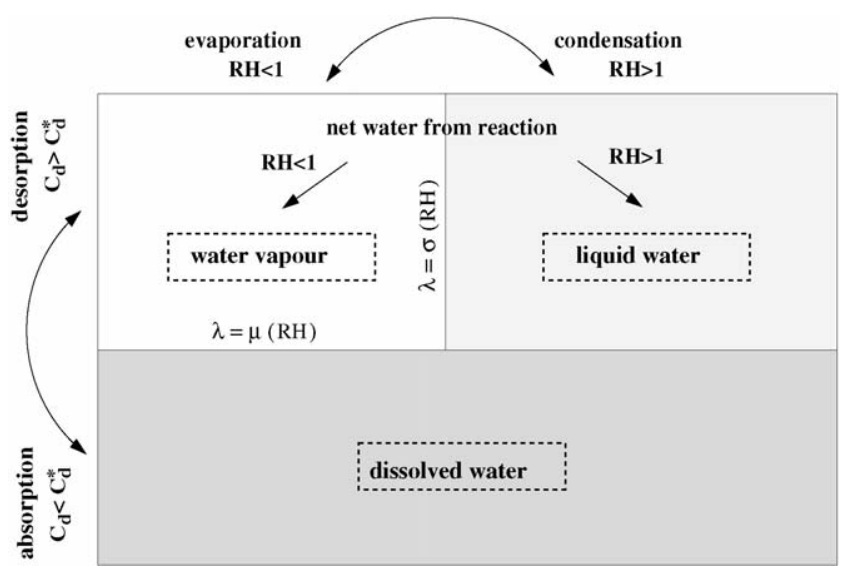

Fig. 3. A schematic representation of the interactions between water in the three phases.

In this equation, $-(\delta s)$ is the entropy associated with ORR, $C_{1}^{\mathrm{v}}$ is the water vapour concentration, $n_{\mathrm{e}}=4$ is the number of electrons transferred in one reaction, $h_{\mathrm{pc}}$ is a mass transfer coefficient, defined below, $h_{\mathrm{gl}}$ is the liquid-gas enthalpy change, $\rho_{\mathrm{l}}$ is the density of liquid water, $C_{1}$ is the specific heat capacity of liquid water, and $k$ is the effective thermal conductivity, found from a volume average of the individual conductivities. For simplicity, we use constant values for $k$. Finally, the quantity $v_{1}$ is the liquid-water interstitial velocity, which we define later.

\subsubsection{Water in the catalyst layer}

The schematic in Fig. 3 depicts the features of the liquidwater model and the assumptions we make in deriving it. For a detailed description of liquid-water transport in MEA we refer to Nam and Kaviany [32].

The mass balance for water dissolved in the electrolyte is written as follows:

$-\frac{\mathrm{d}}{\mathrm{d} x}(\epsilon_{\mathrm{e}} D_{\mathrm{d}} \frac{\mathrm{d} C_{\mathrm{d}}}{\mathrm{d} x}+\underbrace{\frac{5}{44 F v} \lambda \epsilon_{\mathrm{e}} \sigma_{\mathrm{e}} \frac{\mathrm{d} \phi}{\mathrm{d} x}}_{\text {drag }})=-\underbrace{h_{\mathrm{dv}}\left(C_{\mathrm{d}}-C_{\mathrm{d}}^{*}\right)}_{\text {vapour } \leftrightarrow \text { dissolved }}$,

where $D_{\mathrm{d}}$ is the diffusion coefficient for dissolved water. The water vapour mass balance is expressed as follows:

$$
\begin{aligned}
& -\frac{\mathrm{d}}{\mathrm{d} x}\left((1-s) D_{\mathrm{v}} \frac{\mathrm{d} C_{1}^{\mathrm{v}}}{\mathrm{d} x}\right)=-\underbrace{h_{\mathrm{pc}}\left(R T C_{\mathrm{v}}-P_{\mathrm{sat}}\right.}_{\text {liquid } \leftrightarrow \mathrm{gas}}) \\
& +h_{\mathrm{dv}} v\left(C_{\mathrm{d}}-C_{\mathrm{d}}^{*}\right)+\underbrace{\frac{3 \Gamma}{2} \mathcal{R}\left(\eta_{\mathrm{c}}, T, C_{\mathrm{e}}^{\mathrm{s}}\right)}_{\mathrm{H}_{2} \text { O production }},
\end{aligned}
$$

where $D_{\mathrm{v}}$ is the vapour diffusion coefficient. The switch function $\Gamma$ satisfies:

$\Gamma=\left\{\begin{array}{ll}0 & a_{\mathrm{w}}>1 \\ 1 & a_{\mathrm{w}} \leq 1\end{array}\right.$.

The bulk diffusivity $D_{\mathrm{v}}$ is obtained from Chapman-Enskog theory and a Bruggeman correction, and $D_{\mathrm{d}}$ from the relationship 
provided in [42] (with temperature and pressure approximated by their values at the cathode channel):

$D_{\mathrm{d}}=3.5 \times 10^{-5} \lambda \mathrm{e}^{-2436 / T}, \quad D_{\mathrm{v}}=4.1 \times 10^{-9} \frac{T^{3 / 2}}{P} \epsilon_{\mathrm{p}}^{3 / 2}$.

For liquid water, the mass balance is

$-\underbrace{\frac{\mathrm{d}}{\mathrm{d} x}\left(\frac{\epsilon_{\mathrm{p}} \rho_{\mathrm{l}}}{W_{1}} v_{\mathrm{l}}\right)}_{\text {capillary action }}=-h_{\mathrm{pc}}\left(R T C_{\mathrm{v}}-P_{\mathrm{sat}}\right)+\underbrace{\frac{3(\Gamma-1)}{2} \mathcal{R}\left(\eta_{\mathrm{c}}, T, C_{\mathrm{e}}^{\mathrm{s}}\right)}_{\mathrm{H}_{2} \mathrm{O} \text { production }}$,

where $v_{1}$ and $W_{1}$ are respectively the interstitial velocity and molar mass of liquid water. $v_{1}$ is determined by the Darcy-law approximation to momentum conservation:

$v_{1}=\frac{\kappa_{1}}{\mu_{1}} \frac{\mathrm{d} p}{\mathrm{~d} x}$,

where $\kappa_{1}, \mu_{1}$ and $p$ are respectively the relative permeability, viscosity and pressure of the liquid. The latter is given by $p=$ $P-p_{\mathrm{c}}$, so that differentiating Eq. (18) and using Eq. (19) yields:

$-\frac{\rho_{\mathrm{l}}}{\mu_{1} W_{1}} \frac{\mathrm{d}}{\mathrm{d} x}\left(\epsilon_{\mathrm{p}} \kappa_{1}(s)\left[-\frac{\mathrm{d} p_{\mathrm{c}}}{\mathrm{d} s} \frac{\mathrm{d} s}{\mathrm{~d} x}+\frac{\mathrm{d} P}{\mathrm{~d} x}\right]\right)=-h_{\mathrm{pc}}\left(R T C_{\mathrm{v}}-P_{\mathrm{sat}}\right)$

$+\frac{3(\Gamma-1)}{2} \mathcal{R}\left(\eta_{\mathrm{c}}, T, C_{\mathrm{e}}^{\mathrm{s}}\right)$.

There is a great deal of debate surrounding both the form and magnitude of the permeability and capillary pressure. Mazumder and Cole [4], compare the Leverett model employed in [5] with the empirical relationship in [9], in which both of these quantities assume entirely different forms, yielding markedly different predicted levels of saturation. There are also other models of liquid transport in porous media, of varying degrees of sophistication. For example, Nam and Kaviany, [32], scale saturation against an immobile value (below which the liquid water is discontinuous and therefore does not move), and place the scaled saturation inside the Leverett function. All of the forms mentioned can be easily implemented in our model but we choose to follow that in $[4,30,33]$ (and references therein).

$p_{\mathrm{c}}=\sigma_{\mathrm{c}} \mathcal{J}(s), \quad \kappa_{1}(s)=\kappa_{\mathrm{c}} s^{3}$,

where $\kappa_{\mathrm{c}}$ is the absolute permeability of the CCL and $\mathcal{J}(s)$ is the capillary-pressure function. The quantity $\sigma_{\mathrm{c}}$ is defined as follows:

$\sigma_{\mathrm{c}}=\sigma^{\prime} \cos \theta_{\mathrm{c}}^{\mathrm{c}} \sqrt{\frac{\epsilon_{\mathrm{p}}}{\kappa_{\mathrm{c}}}}$

where $\theta_{\mathrm{c}}^{\mathrm{c}}$ is the contact angle for the $\operatorname{CCL}\left(0<\theta_{\mathrm{c}}^{\mathrm{c}}<\pi / 2\right)$ and $\sigma^{\prime}$ is the surface tension. The grouping $2 \sqrt{\epsilon_{\mathrm{p}} / \kappa_{\mathrm{c}}}$ is then equal to the characteristic capillary radius.

The Leverett function for a hydrophilic medium, for which the wetting phase is the liquid, is given by

$\mathcal{J}(s)=1.417(1-s)-2.12(1-s)^{2}+1.262(1-s)^{3}$.

The liquid-vapour phase-change term, derived from that given in [4] and references therein, is driven by the deviation from equilibrium, $R T C_{\mathrm{v}}-P_{\text {sat }}$, where $P_{\text {sat }}$ is the saturation pressure of water and the first term is equivalent to the partial pressure of the vapour. The latter is defined by $X_{\mathrm{V}} P$, where $P$ is the pressure of the gas phase and $X_{\mathrm{V}}$ is the molar fraction of vapour. Using the ideal gas law, $P=C R T, X_{\mathrm{v}}$ is given by

$X_{\mathrm{v}}=\frac{C_{\mathrm{v}}}{C}=\frac{C_{\mathrm{v}} R T}{P}$,

where $C=C_{\mathrm{v}}+C_{\mathrm{N}}+C_{\mathrm{p}}$ is the molar density of the gas. A relationship for the saturation pressure is provided in the discussion of the boundary conditions. In order to distinguish between evaporation and condensation, the mass-transfer coefficient $h_{\mathrm{pc}}$ depends on the sign of the driving force $R T C_{\mathrm{v}}-P_{\text {sat }}$ and its precise form is

$h_{\mathrm{pc}}=\left\{\begin{array}{ll}\kappa_{\mathrm{c}} \frac{\epsilon_{\mathrm{p}}(1-s) X_{\mathrm{v}}}{R T} & R T C_{\mathrm{v}}>P_{\mathrm{sat}} \\ \kappa_{\mathrm{e}} \frac{\epsilon_{\mathrm{p}} s \rho_{\mathrm{l}}}{W_{\mathrm{l}}} & R T C_{\mathrm{v}}<P_{\mathrm{sat}}\end{array}\right.$,

where $\kappa_{\mathrm{c}}$ and $\kappa_{\mathrm{e}}$ are the condensation and evaporation rate constants. The values of $k_{\mathrm{e}}$ and $k_{\mathrm{c}}$ are taken from [2]. We use a continuously differentiable implementation of the following form:

$$
\begin{aligned}
h_{\mathrm{pc}}= & \frac{\kappa_{\mathrm{c}} \epsilon_{\mathrm{p}}(1-s) X_{\mathrm{v}}}{2 R T}\left(1+\frac{\left|R T C_{\mathrm{v}}-P_{\mathrm{sat}}\right|}{R T C_{\mathrm{v}}-P_{\mathrm{sat}}}\right) \\
& +\frac{\kappa_{\mathrm{e}} \epsilon_{\mathrm{p}} s \rho_{1}}{2 W_{1}}\left(1-\frac{\left|R T C_{\mathrm{v}}-P_{\mathrm{sat}}\right|}{R T C_{\mathrm{v}}-P_{\mathrm{sat}}}\right) .
\end{aligned}
$$

In a similar fashion, the vapour-dissolved phase-change term in Eqs. (14) and (15) is driven by the deviation from equilibrium, $C_{\mathrm{d}}-C_{\mathrm{d}}^{*}$, where $C_{\mathrm{d}}^{*}$ is the concentration of dissolved water in equilibrium with vapour. The latter is derived from the following law for the equilibrium water content measured at $80^{\circ} \mathrm{C}$ (found in[43]):

$\lambda^{*}=0.3+10.8 a_{\mathrm{w}}-16 a_{\mathrm{w}}^{2}+14.1 a_{\mathrm{w}}^{3}$,

$C_{\mathrm{d}}^{*}=\frac{\lambda^{*}}{1+0.0126 \lambda^{*}}$,

where $a_{\mathrm{w}}$ is the water vapour activity. To distinguish between desorption and water-uptake of the electrolyte (absorption), the mass-transfer coefficient $h_{\mathrm{dv}}$ depends on the sign of the driving force $C_{\mathrm{d}}-C_{\mathrm{d}}^{*}$. From the experimental and numerical results in [44], we can approximate the coefficients of absorption and desorption as follows:

$h_{\mathrm{dv}}=\left\{\begin{array}{l}\kappa_{\mathrm{a}}(1-s) \lambda C_{\mathrm{d}}-C_{\mathrm{d}}^{*}<0 \\ \kappa_{\mathrm{d}}(1-s) \lambda C_{\mathrm{d}}-C_{\mathrm{d}}^{*}>0\end{array}\right.$,

where $\kappa_{\mathrm{d}}$ and $\kappa_{\mathrm{e}}$ are given in Table 1 . Their weak dependence on temperature variations is neglected. The factor $1-s$ accounts for the blockage of the pores by the liquid water. To implement this approach we use a continuously differentiable form of

$$
\begin{aligned}
h_{\mathrm{dv}}= & \kappa_{\mathrm{d}}(1-s) \lambda\left(1+\frac{\left|C_{\mathrm{d}}-C_{\mathrm{d}}^{*}\right|}{C_{\mathrm{d}}-C_{\mathrm{d}}^{*}}\right) \\
& +\kappa_{\mathrm{d}}(1-s) \lambda\left(1-\frac{\left|C_{\mathrm{d}}-C_{\mathrm{d}}^{*}\right|}{C_{\mathrm{d}}-C_{\mathrm{d}}^{*}}\right),
\end{aligned}
$$


Table 1

A list of model parameter values

\begin{tabular}{|c|c|c|c|}
\hline Symbol & Quantity & Size & References \\
\hline$L$ & Catalyst layer thickness & $22.5 \mu \mathrm{m}$ & \\
\hline$L_{\mathrm{m}}$ & Membrane thickness & $62.5 \mu \mathrm{m}$ & \\
\hline$L_{\mathrm{G}}$ & Cathode GDL thickness & $200 \mu \mathrm{m}$ & \\
\hline$T_{\mathrm{a}}\left(T_{\mathrm{c}}\right)$ & Anode (cathode) channel temperature & $70^{\circ} \mathrm{C}$ & \\
\hline$a_{\mathrm{w}, \mathrm{c}}\left(a_{\mathrm{w}, \mathrm{a}}\right)$ & Cathode (anode) channel water activity & $1(1)$ & \\
\hline $\bar{C}$ & Total gas concentration in cathode channel & $P_{\mathrm{c}} /\left(R T_{\mathrm{c}}\right) \mathrm{mol} \mathrm{m}^{-3}$ & \\
\hline $\bar{C}_{\mathrm{p}}$ & Oxygen concentration in cathode channel & $0.05 \bar{C} \mathrm{~mol} \mathrm{~m}^{-3}$ & \\
\hline$P_{\mathrm{c}}$ & Gas pressure in the cathode channel & 30 psig $(206.842 \mathrm{kPa})$ & \\
\hline$\epsilon_{\mathrm{p}}$ & Volume fraction of primary pores & 0.6 & {$[10-12]$} \\
\hline$\epsilon_{\mathrm{e}}^{i}$ & Electrolyte volume fraction in agglomerates & 0.2 & {$[10-12]$} \\
\hline$\epsilon_{\mathrm{a}}$ & Volume fraction of $\mathrm{C}, \mathrm{Pt}$ and small pores & 0.3 & {$[10-12]$} \\
\hline$\epsilon_{\mathrm{g}}$ & Porosity of the GDL & 0.75 & {$[49]$} \\
\hline$c_{\mathrm{O}_{2}, \text { ref }}$ & Reference oxygen concentration & $4.55 \mathrm{~mol} \mathrm{~m}^{-3}$ & {$[6]$} \\
\hline$D_{\mathrm{l}}$ & Oxygen diffusivity in liquid water $\left(60^{\circ} \mathrm{C}\right)$ & $4.82 \times 10^{-9} \mathrm{~m}^{2} \mathrm{~s}^{-1}$ & [37] \\
\hline$\alpha_{\mathrm{c}}$ & Cathodic transfer coefficient & 0.55 & \\
\hline$\alpha_{\mathrm{a}}$ & Anodic transfer coefficient & 0.45 & \\
\hline$E_{0}$ & Open circuit potential & $0.95 \mathrm{~V}$ & \\
\hline$D_{\mathrm{ag}}$ & Agglomerate diameter $=2 R_{\mathrm{ag}}$ & $0.4 \mu \mathrm{m}$ & {$[10-12]$} \\
\hline$\delta_{0}$ & Electrolyte film thickness without swelling & $15 \mathrm{~nm}$ & {$[17]$} \\
\hline$N$ & Number of agglomerates per unit volume & $1.257 \times 10^{19} \mathrm{~m}^{-3}$ & Est. \\
\hline$a_{\mathrm{pt}}$ & Specific surface area of $\mathrm{Pt}$ & $1000 \mathrm{~cm}^{2}(\mathrm{mg} \mathrm{Pt})^{-1}$ & {$[50]$} \\
\hline$m_{\mathrm{pt}}$ & Pt loading & $0.4(\mathrm{mg} \mathrm{Pt}) \mathrm{cm}^{-2}$ & {$[3]$} \\
\hline$h_{\mathrm{pe}}$ & Oxygen mass transfer rate & $10^{5} \mathrm{~s}^{-1}$ & Est. \\
\hline$H$ & $\mathrm{O}_{2}$ Henry's law constant (dimensionless) & 0.3 & {$[51]$} \\
\hline$\kappa_{\mathrm{a}}\left(\kappa_{\mathrm{d}}\right)$ & Absorption (desorption) constant & $10^{-5} / 9\left(10^{-5} / 3\right) \mathrm{m} \mathrm{s}^{-1}$ & {$[2]$} \\
\hline$\theta_{\mathrm{c}}^{\mathrm{c}}\left(\theta_{\mathrm{c}}^{\mathrm{g}}\right)$ & CCL (GDL) contact angle & $90^{\circ}\left(120^{\circ}\right)$ & $([33])$ \\
\hline$\kappa_{\mathrm{c}}\left(\kappa_{\mathrm{g}}\right)$ & Absolute permeability of CCL (GDL) & $10^{-13}\left(8.7 \times 10^{-12}\right) \mathrm{m}^{2}$ & {$[52]([49])$} \\
\hline$\mu_{1}$ & Liquid-water viscosity & $10^{-3} \mathrm{Kg} \mathrm{m}^{-1} \mathrm{~s}^{-1}$ & {$[4]$} \\
\hline$\sigma^{\prime}$ & Surface tension & $0.07 \mathrm{~N} \mathrm{~m}^{-1}$ & {$[4]$} \\
\hline$k$ & Thermal conductivity of CCL & $2 \mathrm{~W} \mathrm{~m}^{-1} \mathrm{~K}^{-1}$ & [29] \\
\hline$k_{\mathrm{m}}$ & Thermal conductivity of membrane & $1 \mathrm{~W} \mathrm{~m}^{-1} \mathrm{~K}^{-1}$ & [29] \\
\hline$k_{\mathrm{G}}$ & Thermal conductivity of GDL & $4 \mathrm{~W} \mathrm{~m}^{-1} \mathrm{~K}^{-1}$ & {$[29]$} \\
\hline$\Omega$ & Liquid-water removal constant & $0.075 \mathrm{~m}^{-1}$ & Fitted \\
\hline$i_{\mathrm{O}_{2}, \mathrm{c}}$ & Reference cathode exchange current density & $40 \mathrm{~A} \mathrm{~m}^{-2}$ & Fitted \\
\hline$\rho_{\mathrm{l}} C_{1}$ & Heat capacitance of water & $4.184 \times 10^{6} \mathrm{~J} \mathrm{~m}^{-3} \mathrm{~K}^{-1}$ & Est. \\
\hline$-\delta s$ & Entropy associated with ORR & $162.2 \mathrm{~J} \mathrm{~mol}^{-1} \mathrm{~K}^{-1}$ & {$[53]$} \\
\hline
\end{tabular}

\subsubsection{Schroeder's paradox}

It is well known that the water content $\lambda$ depends on the water activity, $a_{\mathrm{w}}=X_{\mathrm{v}} P / P_{\text {sat }}$. The precise relationship was correlated by Hinatsu et al. in [43],

$\lambda_{\mathrm{v}}=\lambda_{\mathrm{a}}=0.3+10.8 a_{\mathrm{w}}-16 a_{\mathrm{w}}^{2}+14.1 a_{\mathrm{w}}^{3} \quad($ at $353 \mathrm{~K})$,

which has a maximum of $\lambda_{\mathrm{v}}=9.1$ at equilibrium with vapour. However, when the electrolyte is submerged in liquid water its equilibrium water content appears to jump discontinuously to a higher value, which, following [44], we take to be $\lambda=16.8$.

In an attempt to capture this anomaly, known as Schroeder's paradox, we define a critical saturation, $s_{*}$, at which the agglomerates are entirely coated with liquid water and equilibrium with liquid water is achieved. This critical value is approximated as the immobile saturation, below which the water mass is not continuous (and therefore does not flow). We point out that this is an approximation, based on the assumption that the electrolyte is continuously covered by water for $s>s_{*}$. We use the value of $s_{*}=0.1$ given in [32], and refer to that paper for references related to its measurement. The water content at the boundary between the CCL and membrane is kept continuous by enforc- ing equilibrium between the membrane and liquid water when $s\left(L_{2}\right)=s_{*}$. Alternatively, we may assume that the water content in the membrane drops continuously to a vapour-equilibrated value at $x=L_{3}$, but this does not qualitatively alter the results. We therefore make the simpler choice and avoid further assumptions in specifying the rate of decrease.

A list of parameters and their values can be found in Table 1.

\subsection{Reaction rate and limiting current density}

The ORR rate (in mol $\mathrm{m}^{-3} \mathrm{~s}^{-1}$ ) is given by the Butler-Volmer law and first-order kinetics in oxygen concentration:

$\mathcal{R}\left(\eta_{\mathrm{c}}, T, C_{\mathrm{e}}^{\mathrm{s}}\right)=\frac{a i_{\mathrm{O}_{2}, \mathrm{c}}}{F c_{\mathrm{O}_{2}, \text { ref }}} \epsilon_{\mathrm{e}} C_{\mathrm{e}}^{\mathrm{s}}\left(\mathrm{e}^{\alpha_{\mathrm{a}} F \eta_{\mathrm{c}} / R T}-\mathrm{e}^{-\alpha_{\mathrm{c}} F \eta_{\mathrm{c}} / R T}\right)$,

where $i_{\mathrm{O}_{2}, \mathrm{c}}$ is the cathode exchange current density, $\alpha_{\mathrm{a}}$ and $\alpha_{\mathrm{c}}$ are the anodic and cathodic transfer coefficients, $c_{\mathrm{O}_{2}}$, ref is a reference oxygen molar concentration, $a$ is the surface area of catalyst per unit volume of catalyst layer, $\eta_{\mathrm{c}}$ is the overpotential and $R$ is the universal gas constant $\left(8.314 \mathrm{~J} \mathrm{~mol}^{-1} \mathrm{~K}^{-1}\right)$ ). The quantity $a$ is a function of the specific $\mathrm{Pt}$ surface area (Pt surface area per unit mass of Platinum), $a_{\mathrm{pt}}$, the Pt loading, $m_{\mathrm{pt}}$, and the CCL 
thickness, $L$ :

$a=a_{\mathrm{pt}} m_{\mathrm{pt}} / L$.

However, the active platinum surface area is much more difficult to characterize. The overpotential, $\eta_{\mathrm{c}}$, is defined precisely through the relationship:

$\eta_{\mathrm{c}}=U_{\mathrm{c}}-E_{0}-\phi$,

where $U_{\mathrm{c}}$ is the potential of the carbon matrix (constant by assumption) and $E_{0}$ is the so-called open circuit potential, assumed constant. The value of oxygen concentration in the ButlerVolmer expression, (31), ought to reflect accurately that reaction takes place at the surfaces of the carbon agglomerates. The concentration at these surfaces, $C_{\mathrm{e}}^{\mathrm{s}}$, is generally different from the bulk value in the electrolyte, particularly given the restricted diffusion of oxygen. $C_{\mathrm{e}}^{\mathrm{s}}$ can be related to the bulk value, $C_{\mathrm{e}}$, by balancing the rate of reaction with the rate of diffusion of oxygen to the surface of the agglomerates (at steady state). This mass balance can be approximated as follows:

$\gamma^{\prime}\left(C_{\mathrm{e}}-C_{\mathrm{e}}^{\mathrm{s}}\right)=\frac{1}{4} \mathcal{R}\left(\eta_{\mathrm{c}}, T, C_{\mathrm{e}}^{\mathrm{s}}\right)$,

where $\gamma^{\prime}$ is the rate of oxygen diffusion through the electrolyte/water film to the surface of the agglomerates (in $\mathrm{s}^{-1}$ ). Using the definition of $\mathcal{R}\left(\eta_{\mathrm{c}}, T, C_{\mathrm{e}}^{S}\right)$ and solving the resulting equation for $C_{\mathrm{e}}^{\mathrm{s}}$ then yields:

$C_{\mathrm{e}}^{\mathrm{s}}=\frac{\gamma^{\prime} C_{\mathrm{e}}}{\gamma^{\prime}+\epsilon_{\mathrm{e}} r\left(\mathrm{e}^{\alpha_{\mathrm{a}} F \eta_{\mathrm{c}} / R T}-\mathrm{e}^{-\alpha_{\mathrm{c}} F \eta_{\mathrm{c}} / R T}\right)} \quad$ where

$r=\frac{a i_{\mathrm{O}_{2}, \mathrm{c}}}{4 F c_{\mathrm{O}_{2}, \mathrm{ref}}}$

so that the final form of the reaction rate is

$$
\begin{aligned}
\mathcal{R}\left(\eta_{\mathrm{c}}, T, C_{\mathrm{e}}^{\mathrm{s}}\right) & \equiv \mathcal{R}\left(\eta_{\mathrm{c}}, T, C_{\mathrm{e}}\right) \\
& =\frac{\gamma^{\prime} \epsilon_{\mathrm{e}} r C_{\mathrm{e}}\left(\mathrm{e}^{\alpha_{\mathrm{a}} F \eta_{\mathrm{c}} / R T}-\mathrm{e}^{-\alpha_{\mathrm{c}} F \eta_{\mathrm{c}} / R T}\right)}{\gamma^{\prime}+\epsilon_{\mathrm{e}} r\left(\mathrm{e}^{\alpha_{\mathrm{a}} F \eta_{\mathrm{c}} / R T}-\mathrm{e}^{-\alpha_{\mathrm{c}} F \eta_{\mathrm{c}} / R T}\right)} .
\end{aligned}
$$

In order to relate the diffusion rate $\gamma^{\prime}$ to the microscopic properties of the CCL, we define an electrolyte film thickness, $\delta_{\mathrm{e}}$, an agglomerate radius, $R_{\mathrm{ag}}$, and the number of agglomerates per unit volume, $N$ :

$$
\gamma^{\prime}\left(C_{\mathrm{e}}-C_{\mathrm{e}}^{\mathrm{s}}\right) \approx A \underbrace{\frac{D_{\mathrm{e}}}{\delta_{\mathrm{e}}}\left(C_{\mathrm{e}}-C_{\mathrm{e}}^{\mathrm{s}}\right)}_{\text {molar flux }} \quad \text { so that } \quad \gamma^{\prime}=\frac{A D_{\mathrm{e}}}{\delta_{\mathrm{e}}},
$$

where $A$ is the specific surface area of agglomerates (we assume that all of the surface area is covered, whereupon $\left.A=4 \pi R_{\mathrm{ag}}^{2} N\right)$. The volume of electrolyte attached to the surface of each agglomerate is $\epsilon_{\mathrm{e}}^{\mathrm{f}} / N$, which, from our assumptions, covers the entire surface of the agglomerate. The thickness $\delta_{0}^{\mathrm{e}}$ (without swelling) and $\epsilon_{0}^{\mathrm{f}}$ are therefore related as follows:

$\delta_{0}^{\mathrm{e}}=\left(R_{\mathrm{ag}}^{3}+\frac{3 \epsilon_{0}^{f}}{4 \pi N}\right)^{1 / 3}-R_{\mathrm{ag}}$.
The electrolyte swelling results in a volume change equal to (per agglomerate) $\left(\epsilon_{\mathrm{e}}^{\mathrm{f}}-\epsilon_{0}^{\mathrm{f}}\right) / N$. The electrolyte film thickness is then given by

$\delta_{\mathrm{e}}=\left(\left(R_{\mathrm{ag}}+\delta_{0}^{\mathrm{e}}\right)^{3}+\frac{3\left(\epsilon_{\mathrm{e}}^{\mathrm{f}}-\epsilon_{0}^{\mathrm{f}}\right)}{4 \pi N}\right)^{1 / 3}-R_{\mathrm{ag}}$.

When $s>s_{*}$, as previously defined, the liquid water present in the catalyst layer will provide additional resistance to the oxygen, with a different diffusion coefficient $D_{1}$. Given the hydrophilic nature of the electrolyte, we assume that any liquid water coats the entire surface of the agglomerates. Based on these assumptions, the thickness of the water layer is given by

$\delta_{1}=\left(\left(R_{\mathrm{ag}}+\delta_{\mathrm{e}}\right)^{3}+\frac{3 s \epsilon_{\mathrm{p}}}{4 \pi N}\right)^{1 / 3}-R_{\mathrm{ag}}$,

with a total film thickness

$\delta=\delta_{\mathrm{e}}+\delta_{1}$

The quantity $\gamma^{\prime}$ then has to be modified; it is decomposed into a term arising from diffusion through the water layer, $\gamma_{1}^{\prime}$, and a term of the type described above, $\gamma_{\mathrm{e}}^{\prime}$. To approximate the concentration of oxygen at the agglomerate surfaces two balances need to be performed, one for diffusion through the water, to relate the bulk concentration to concentration at the water/electrolyte interface, and one between diffusion through the electrolyte and reaction, to relate the latter concentration to $C_{\mathrm{e}}^{\mathrm{s}}$. The reaction rate then takes the form (35) with

$$
\begin{aligned}
& \gamma^{\prime}=\frac{\gamma_{1}^{\prime} \gamma_{\mathrm{e}}^{\prime}}{\gamma_{1}^{\prime}+\gamma_{\mathrm{e}}^{\prime}}, \quad \text { where } \quad \gamma_{1}^{\prime}=\frac{A^{\prime} D_{\mathrm{l}}}{\delta_{\mathrm{l}}}, \\
& \gamma_{\mathrm{e}}^{\prime}=\frac{A D_{\mathrm{e}}}{\delta_{\mathrm{e}}}, \quad A^{\prime}=4 \pi\left(R_{\mathrm{ag}}+\delta_{\mathrm{e}}\right)^{2} N .
\end{aligned}
$$

The Henry constant $H$ would likewise require modification. It is approximately 0.3 for water under typical operating conditions, and was assigned the value 0.15 for Nafion in [27]. For simplicity, we assume the water-based value throughout and approximate it as a constant.

Note that the quantity $N$ is a function of both the radius of the agglomerates and their distribution, for example, the more densely packed the agglomerates the larger $N$.

The current flow is the reverse of the $\mathrm{H}_{3} \mathrm{O}^{+}$flow, so that the current density, $I^{\prime} \mathrm{A} \mathrm{m}^{-2}$, is

$I^{\prime}=4 F r \int_{0}^{L} \frac{\gamma^{\prime} \epsilon_{\mathrm{e}} C_{\mathrm{e}}\left(\mathrm{e}^{\alpha_{\mathrm{a}} F \eta_{\mathrm{c}} / R T}-\mathrm{e}^{-\alpha_{\mathrm{c}} F \eta_{\mathrm{c}} R T}\right)}{\gamma^{\prime}+\epsilon_{\mathrm{e}} r\left(\mathrm{e}^{\alpha_{\mathrm{a}} F \eta_{\mathrm{c}} / R T}-\mathrm{e}^{-\alpha_{\mathrm{c}} F \eta_{\mathrm{c}} / R T}\right)} \mathrm{d} x$.

\subsection{Membrane}

We now describe the equations employed in the membrane, $L_{2}<x<L_{3}$, within which we assume that oxygen and vapour do not exist, i.e., the membrane is impenetrable to both. The membrane potential is modelled in a manner similar to the electrolyte potential in the CCL, Eq. (8),

$-\frac{\mathrm{d}}{\mathrm{d} x}\left(\frac{\sigma_{\mathrm{e}}}{F} \frac{\mathrm{d} \phi}{\mathrm{d} x}+D_{\mathrm{H}} C_{\mathrm{d}} \frac{\mathrm{d} C_{\mathrm{H}}}{\mathrm{d} x}\right)=0$, 
where $\sigma_{\mathrm{e}}$ and $D_{\mathrm{H}}$ were previously defined. The movement of water in the membrane is either as a dissolved species or possibly by convection as a liquid, the latter depending on the state of the membrane. It has been postulated by Weber and Newman, [45], that the convective movement increases with increased water content because the membrane walls are inflated by the action of the water. However, as Mazumder demonstrates in [46], the role of convection is typically outweighed by both diffusion and electro-osmotic drag, even when a pressure increase of $2 \mathrm{~atm}$ from anode to cathode is maintained. We therefore neglect it, particularly since we will not consider such substantial pressure drops. The equation for dissolved water is then

$-\frac{\mathrm{d}}{\mathrm{d} x}\left(D_{\mathrm{d}} \frac{\mathrm{d} C_{\mathrm{d}}}{\mathrm{d} x}+\frac{5}{44 F v} \lambda \sigma_{\mathrm{e}} \frac{\mathrm{d} \phi}{\mathrm{d} x}\right)=0$.

The equation for temperature now involves only a conduction term and ohmic losses

$-\frac{\mathrm{d}}{\mathrm{d} x}\left(k_{\mathrm{m}} \frac{\mathrm{d} T}{\mathrm{~d} x}\right)=\sigma_{\mathrm{e}}\left(\frac{\mathrm{d} \phi}{\mathrm{d} x}\right)^{2}$,

where $k_{\mathrm{m}}$ is the effective thermal conductivity of the membrane. At the interface between the membrane and CCL a further condition is enforced to ensure the continuity of the water content, $\lambda$. This condition was discussed above.

\subsection{Gas diffusion layer}

In the GDL, $0<x<L_{1}$, we specify balances for the masses of oxygen, nitrogen, vapour and liquid water, together with an energy balance. Equations for the first two are

$-\frac{\mathrm{d}}{\mathrm{d} x}\left(D_{\mathrm{p}}^{\mathrm{G}}(1-s) \frac{\mathrm{d} C_{\mathrm{p}}}{\mathrm{d} x}\right)=-\frac{\mathrm{d}}{\mathrm{d} x}\left(D_{\mathrm{N}}^{\mathrm{G}}(1-s) \frac{\mathrm{d} C_{\mathrm{N}}}{\mathrm{d} x}\right)=0$,

where $D_{\mathrm{p}}^{\mathrm{G}}$ and $D_{\mathrm{N}}^{\mathrm{G}}$ are the effective molecular diffusion coefficients for oxygen and nitrogen in the GDL, and $\epsilon_{\mathrm{g}}$ is the GDL porosity.

The equation for vapour concentration is

$-\frac{\mathrm{d}}{\mathrm{d} x}\left((1-s) D_{\mathrm{v}}^{\mathrm{G}} \frac{\mathrm{d} C_{1}^{\mathrm{v}}}{\mathrm{d} x}\right)=-h_{\mathrm{pc}}\left(R T C_{\mathrm{v}}-P_{\mathrm{sat}}\right)$,

where $D_{\mathrm{v}}^{\mathrm{G}}$ is the effective molecular diffusion coefficient for vapour in the GDL. $D_{\mathrm{p}}^{\mathrm{G}}, D_{\mathrm{N}}^{\mathrm{G}}$ and $D_{\mathrm{v}}^{\mathrm{G}}$ are approximated as in (4) and (17) (with $\epsilon_{\mathrm{p}}$ replaced with $\epsilon_{\mathrm{g}}$ ), evaluated at the cathode channel conditions. The phase-change term on the right-hand side of (47) is defined as in (25) and (26), with $\epsilon_{\mathrm{p}}$ replaced with $\epsilon_{\mathrm{g}}$.

The energy equation balances conduction, convective heat flow arising from the liquid water motion and heating from phase change,

$-\frac{\mathrm{d}}{\mathrm{d} x}\left(k_{\mathrm{G}} \frac{\mathrm{d} T}{\mathrm{~d} x}-s \epsilon_{\mathrm{g}} \rho_{\mathrm{l}} C_{\mathrm{l}} v_{\mathrm{l}} T\right)=h_{\mathrm{g} 1} h_{\mathrm{pc}}\left(R T C_{\mathrm{v}}-P_{\text {sat }}\right)$,

where $k_{\mathrm{G}}$ is the effective thermal conductivity of the GDL.
Finally, the equation for liquid water is

$-\frac{\rho_{\mathrm{l}}}{\mu_{1} W_{1}} \frac{\mathrm{d}}{\mathrm{d} x}\left(\epsilon_{\mathrm{g}} \kappa_{1}(s)\left[-\frac{\mathrm{d} p_{\mathrm{c}}}{\mathrm{d} s} \frac{\mathrm{d} s}{\mathrm{~d} x}+\frac{\mathrm{d} P}{\mathrm{~d} x}\right]\right)=-h_{\mathrm{pc}}\left(R T C_{\mathrm{v}}-P_{\text {sat }}\right)$,

with

$p_{\mathrm{c}}=\sigma_{\mathrm{g}} \mathcal{J}(s), \quad \kappa_{\mathrm{l}}(s)=\kappa_{\mathrm{g}} s^{3}, \quad \sigma_{\mathrm{g}}=\sigma^{\prime} \cos \theta_{\mathrm{c}}^{\mathrm{g}} \sqrt{\frac{\epsilon_{\mathrm{g}}}{\kappa_{\mathrm{g}}}}$,

where $\kappa_{\mathrm{g}}$ is the absolute permeability of the GDL and $\theta_{\mathrm{c}}^{\mathrm{g}}$ is the contact angle $\left(\pi / 2<\theta_{\mathrm{c}}^{\mathrm{g}}<\pi\right)$. The Leverett function for a hydrophobic medium, for which the wetting phase is the gas phase, is given by

$\mathcal{J}(s)=1.417 s-2.12 s^{2}+1.262 s^{3}$.

\subsection{Boundary conditions}

For the discussion of the boundary conditions we recall Fig. 1, which depicts the geometry. At the interface between the membrane and CCL, $x=L_{2}$, the fluxes of oxygen (in the pores), water vapour, nitrogen and liquid water are taken to be zero; that is, these species are assumed not to penetrate the membrane. It is possible that small amounts of the dissolved oxygen reach the anode and small amounts of hydrogen reach the cathode. These are primarily degradation issues, which are dealt with in a forthcoming paper. Since protons have no effective transport mechanism in the GDL, the flux of protons at the interface between the GDL and CCL, $x=L_{1}$, is zero. Similarly, the dissolved-water and oxygen fluxes must be zero at $x=L_{1}$ in order to ensure their continuity:

$$
\begin{gathered}
\frac{\mathrm{d} C_{\mathrm{e}}}{\mathrm{d} x}=D_{\mathrm{d}} \frac{\mathrm{d} C_{\mathrm{d}}}{\mathrm{d} x}+\frac{5}{44 F \nu} \lambda \sigma_{\mathrm{e}} \frac{\mathrm{d} \phi}{\mathrm{d} x}=\frac{\mathrm{d} \phi}{\mathrm{d} x}=0, \quad x=L_{1} \\
\frac{\mathrm{d} C_{\mathrm{p}}}{\mathrm{d} x}=\frac{\mathrm{d} C_{\mathrm{e}}}{\mathrm{d} x}=\frac{\mathrm{d} C_{\mathrm{v}}}{\mathrm{d} x}=\frac{\mathrm{d} s}{\mathrm{~d} x}=0, \quad x=L_{2} .
\end{gathered}
$$

At the interface between the cathode channel and the GDL interface, $x=0$, the oxygen and vapour concentrations are prescribed. At the membrane/ACL interface, $x=L_{3}$, the membrane potential is approximated as zero

$C_{\mathrm{p}}(0)=\bar{C}_{\mathrm{p}}, \quad C_{\mathrm{v}}(0)=\bar{C}_{\mathrm{v}}, \quad \phi\left(L_{3}\right)=0$.

The value of $\bar{C}_{\mathrm{v}}$ is calculated from the water activity of the cathode channel, $a_{\mathrm{w}, \mathrm{c}}$, which is defined as $\bar{X}_{\mathrm{v}} P_{\mathrm{c}} / P_{\mathrm{sat}}$, where $\bar{X}_{\mathrm{v}}$ is the molar fraction of vapour in the cathode channel, $P_{\mathrm{c}}$ is the cathode-channel gas pressure and $P_{\mathrm{sat}, \mathrm{c}}$ is the cathodechannel saturation pressure. The saturation pressure, a function of temperature, is given by the formula in [39]:

$$
\begin{aligned}
\log _{10} P_{\text {sat }}= & -2.1794+0.02953(T-273)-9.1837 \\
& \times 10^{-5}(T-273)^{2}+1.4454 \times 10^{-7}(T-273)^{3} .
\end{aligned}
$$

From these relationships we derive $\bar{C}_{\mathrm{v}}$ as follows:

$\bar{C}_{\mathrm{v}}=\frac{R T_{\mathrm{c}} P_{\mathrm{sat}, \mathrm{c}}}{P_{\mathrm{c}}} \bar{C}$, 
where $\bar{C}$ is the total gas molar concentration in the cathode channel, calculated from the cathode-channel pressure:

$\bar{C}=\frac{P_{\mathrm{c}}}{R T_{\mathrm{c}}}$.

The dissolved-water concentration at the membrane/ACL interface is assumed to be in equilibrium with the vapour-phase concentration if $s\left(L_{2}\right)<s_{*}$, and to be in equilibrium with water if $s\left(L_{2}\right) \geq s_{*}$. The water activity at $x=L_{2}, a_{\mathrm{w}, \mathrm{a}}$, is approximated by the anode-channel value (since water is not produced in the ACL), yielding the following expression for $C_{\mathrm{d}}$ :

$\lambda=\lambda_{\mathrm{a}}=0.3+10.8 a_{\mathrm{w}, \mathrm{a}}-16 a_{\mathrm{w}, \mathrm{a}}^{2}+14.1 a_{\mathrm{w}, \mathrm{a}}^{3}$,

$C_{\mathrm{d}}\left(L_{3}\right)=\frac{\lambda_{\mathrm{a}}}{1+0.0126 \lambda_{\mathrm{a}}}$.

In the cathode channel the temperature is prescribed. The flux of heat at $x=L_{3}$ is approximated using the anode channel temperature $T_{\mathrm{a}}$. We assume that the heat flux is constant across the anode GDL and ACL, yielding:

$T(0)=T_{\mathrm{c}}, \quad k_{\mathrm{m}} \frac{\mathrm{d} T}{\mathrm{~d} x}\left(L_{3}\right)=\frac{k_{\mathrm{G}}}{L_{\mathrm{G}}}\left(T_{\mathrm{a}}-T\left(L_{3}\right)\right)$.

We are assuming the that the anode gas diffusion layer is identical to that on the cathode side. This is not necessarily so and this assumption can easily be relaxed.

The final boundary condition is that for the liquid water at the interface between the cathode channel and the GDL. It is common in modelling studies, for example [3,5-7], to assume either a zero saturation or zero liquid-water flux at this location, or along portions of the channel/GDL interface in two dimensions. In [47], Weng and Wang specify the saturation at the GDL/channel interface, which, as they state, is likely to depend sensitively on the gas flow in the channel, current density and wettability. According to their results, high levels of saturation are possible only if the prescribed value at the boundary is high or the GDL permeability is unrealistically small. In our model, the counterpart to this boundary condition depends on the gas flow and wettability, and is written as a flux. We show that high saturation levels are possible within a broad range of realistic parameter space.

The accumulation and removal of liquid water along the channel is a complicated process. Water is expelled from the GDL through preferential openings and forms droplets attached to the surface. These droplets can grow or coalesce to a form larger droplets comparable in size to the channel dimensions, which results is the formation of a liquid film, [48]. Ultimately, the film is wicked along the channel walls toward the exit. The greater the flow velocity in the channel, the more effective the removal of liquid water. A detailed model of this process, accounting for the surface properties of the GDL, phase change, surface tension, two dimensional gas flow and hydrophilicity of the channel walls, is clearly a challenge in its own right that will not be attempted here. Instead, we approximate the process with the following steady-state flux condition at $x=0$ :

$\frac{\mathrm{d} s}{\mathrm{~d} x}(0)-\Omega s(0)=0$, where $\Omega=0$ corresponds to zero water removal. This form is motivated in two ways. Firstly, there should be no removal at zero saturation. Secondly, the parameter $\Omega$ can be seen naturally as (in an average sense) the reciprocal of a water film thickness on the surface of the GDL. When this thickness approaches zero, the saturation is physically zero, which is expressed by $1 / \Omega \rightarrow 0$ in Eq. (59). The thickness, and therefore $\Omega$, is likely to depend sensitively on the flow rate in the channel, [48] (also by analogy with the theory of Ekman transport in ocean modelling).

\section{Experimental details}

The fuel cell was prepared with carbon fibre paper (GDL), carbon supported Platinum catalyst and Nafion membrane, with a $50 \mathrm{~cm}^{2}$ anode geometric area and a masked cathode with a $4 \mathrm{~cm}^{2}$ geometric area. The MEA and cell hardware were designed to minimize any temperature, pressure and concentration gradients in the cell. The small cathode geometric area ensures minimal heat generation and concentration change along the length of the flow-field channels. To further ensure that there was no concentration change along the length of the flow field, we used a stoichiometry of greater than 60 (compared to a normal operating value of approximately 2). Thus, the design was such that variations were predominantly through the MEA, and therefore one-dimensional. Note also that the flow rate was the same in all cases.

Using a load bank, a series of polarization curves were produced under varying conditions to demonstrate the change in the polarization losses relative to oxygen concentration, water activity and temperature.

The oxygen concentration was varied between $3 \%$ and $5 \%$, balanced in nitrogen. A separate investigation was performed at the same concentrations with oxygen balanced with helium. These results are not discussed here.

Three different water activity conditions were tested at a cell temperature of $70^{\circ} \mathrm{C}: a_{\mathrm{w}}=0.6$ anode $/ a_{\mathrm{w}}=0.7$ cathode, $a_{\mathrm{w}}=$ 0.7 anode $/ a_{\mathrm{w}}=0.8$ cathode, and $a_{\mathrm{w}}=1$ on both anode and cathode. Three different cell temperatures were tested at $a_{\mathrm{w}}=1$ to validate the effect of temperature on performance: 70,45 and $25^{\circ} \mathrm{C}$. Only results pertaining to the first are discussed here.

\section{Numerical results}

\subsection{Finite element solution strategy}

We first outline the solution strategy and the manner in which the results were matched to the experimental data (presented in the next section). The governing equations and boundary conditions laid out above were solved using the finite-element method implemented in FEMLAB. The discretization of the equations was achieved on a uniform grid using quartic Lagrange polynomials, allowing the number of grid points to be kept small (typically 32 or 64). The switch functions were substituted with hyperbolic tanh functions to smooth the discontinuities, a standard procedure. Adaptivity, a feature of FEMLAB, was not required in the calculations, though was occasionally used to obtain smoother plots. 

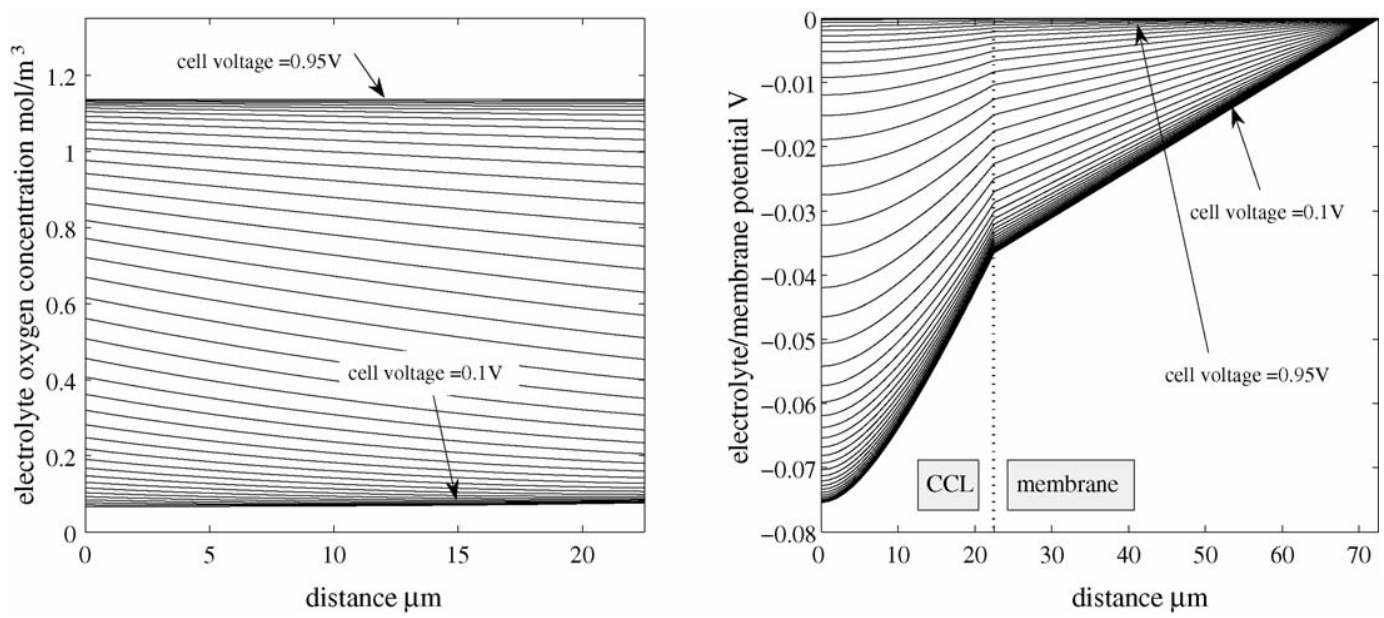

Fig. 4. Electrolyte potential and oxygen concentration profiles corresponding to $a_{\mathrm{w}}=1$ in Fig. 7, as the cell potential is decreased.

In order to compare the modelling with the experimental results, all parameter values were fixed as specified below with the exception of the exchange current density, $i_{\mathrm{O}_{2}, \mathrm{c}}$. The latter was then adjusted to match the experimental data displayed in Figs. 6 and 7. This fitting was required only once, i.e., $i_{\mathrm{O}_{2}, \mathrm{c}}=40 \mathrm{~A} \mathrm{~m}^{-2}$ in both figures. The motivation for this fitting procedure stems from the lack of a consistent value for $i_{\mathrm{O}_{2}, \mathrm{c}}$ in the literature (426 $\mathrm{A} \mathrm{m}^{-2}$ in [41] and $1.5 \times 10^{-4} \mathrm{~A} \mathrm{~m}^{-2}$ in [26]). Similar discrepancies exist in the values of the transfer coefficients and

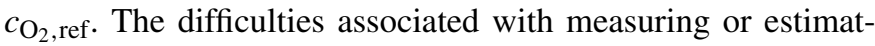
ing these quantities are numerous. For example, $i_{\mathrm{O}_{2}, \mathrm{c}}$ changes from one cathode structure to another (active surface area of platinum), as well as with temperature. The value we derive for $i_{\mathrm{O}_{2}, \mathrm{c}}$ falls well within the broad range of values that appear in the literature. A more detailed discussion of the issues surrounding the measurement of these quantities can be found in [54] and [55].

It should be mentioned that $\Omega$ was fixed at $0.075 \mathrm{~m}^{-1}$. Since a value for $\Omega$ is difficult to determine theoretically, we later present a parametric study of its effect. The flow rate in the cathode channel is assumed to be constant (as in the experiments), which justifies the use of a constant $\Omega$ in all calculations.

\subsection{Experimental validation}

For the modelling results of this section, the parameters values that were used are shown in Table 1, unless otherwise stated.

We begin with the set of results demonstrated in Figs. 4 and 5 , the first of which shows profiles of electrolyte potential and oxygen concentration as the cell voltage (approximated by the carbon potential $U_{\mathrm{c}}$ ) is decreased from 0.95 to $0.1 \mathrm{~V}$. In the calculations, both channels are at $a_{\mathrm{w}}=1$ and $70^{\circ} \mathrm{C}$, and the oxygen concentration in the cathode channel is $5 \%$ of the total. The second figure shows the saturation levels for both the case above and with $a_{\mathrm{w}}=0.7$ anode $/ a_{\mathrm{w}}=0.8$ cathode, indicating the effect that the water activity in the channels has on the flooding of the cathode: that saturation levels can be high at low cell voltage and with high water activity in the channels. We return to this point later.
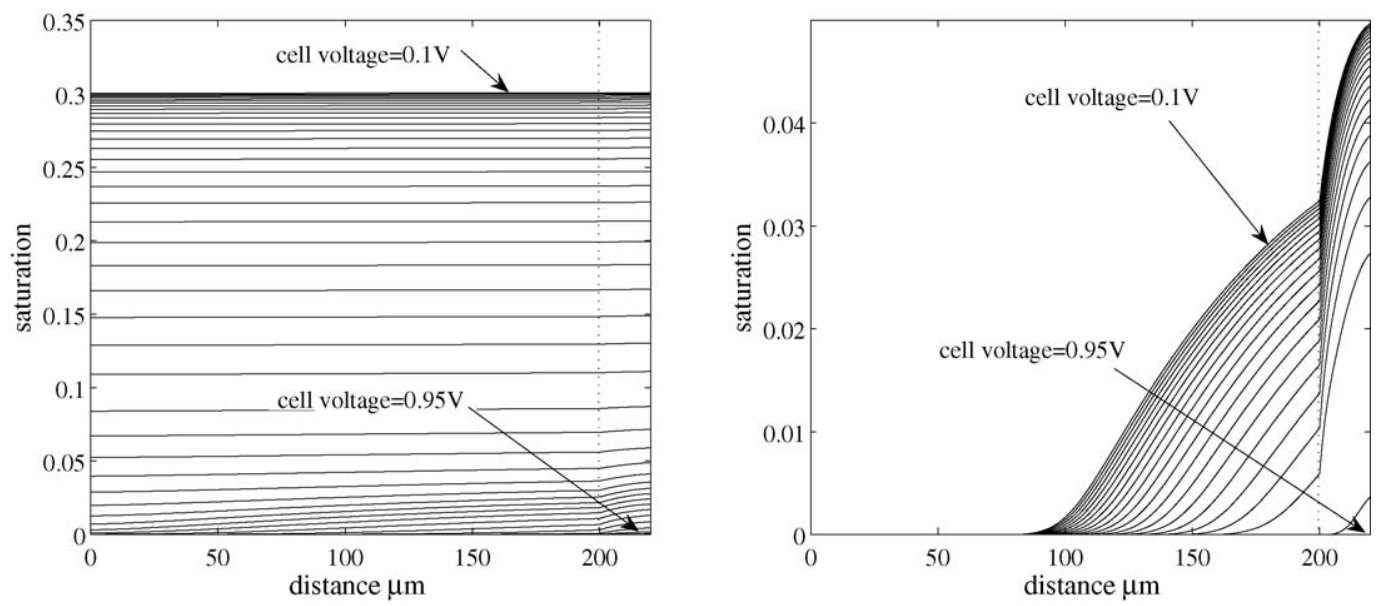

Fig. 5. Saturation levels for decreasing cell voltage corresponding to $a_{\mathrm{w}}=1$ (left) and $a_{\mathrm{w}}=0.7$ anode $/ a_{\mathrm{w}}=0.8$ cathode, the polarization curves for which are given in Fig. 7. 


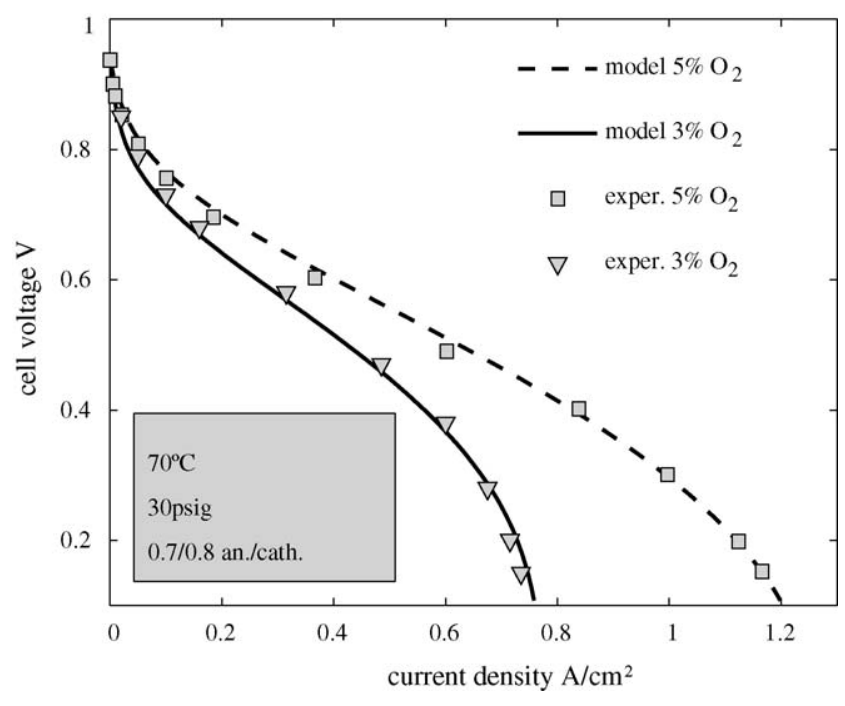

Fig. 6. Comparison of the modelling and experimental polarization curves at 5 and $3 \%$ oxygen concentration (see Table 1 for a full list of parameter values).

To demonstrate the comparison between the modelling results and those of the experimental procedure described in the previous section, we refer to Figs. 6 and 7. The first of these figures shows the polarization curves at two different oxygen concentrations, with $a_{\mathrm{w}}=0.7$ anode $/ a_{\mathrm{w}}=0.8$ cathode. The second figure shows polarization curves at two different values of water activity in the channels, with a $5 \%$ oxygen concentration. In both of these figures, the conditions not specified are as in Table 1, except that the thickness of the CCL is $25 \mu \mathrm{m}$.

The agreement between the modelling results and the experimental data is excellent. The second figure clearly underlines the ability of the model to capture the impact of water on both the membrane and CCL/GDL. The modelling and experimental results strongly suggest that the increased hydration of the membrane at high water activity leads to improved performance at high and mid-range cell voltages. However, as the current den-

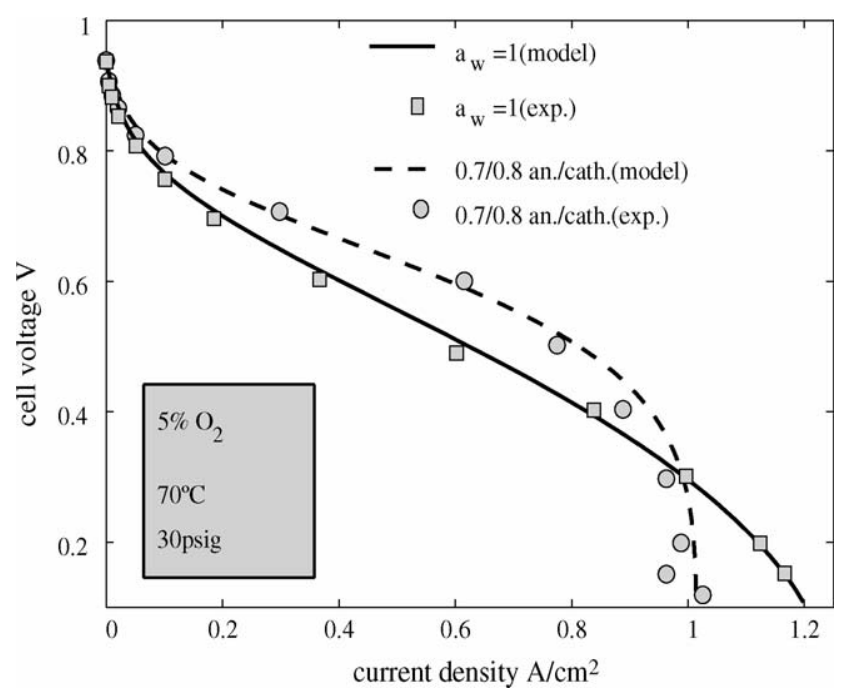

Fig. 7. Comparison of the modelling and experimental polarization curves for two $a_{\mathrm{w}}$ configurations at $5 \%$ concentration of oxygen (see Table 1 for a full list of parameter values). sity increases so does the water generated in the CCL, which leads to significant oxygen diffusion limitation from flooding, and ultimately to a better performance at low water activity. These features are very well captured by the model. Below we say more on the effects of changes in the channel water activity, particularly when coupled to changes in the channel temperatures.

\subsection{Effects of GDL and CCL structures}

The magnitudes of the permeabilities, $\kappa_{\mathrm{c}}$ and $\kappa_{\mathrm{g}}$, and capillary diffusion coefficients, $\sigma_{\mathrm{c}}$ and $\sigma_{\mathrm{g}}$, are subject to a great deal of uncertainty. The effect that changes in these quantities have on the flooding behaviour, and therefore performance, are not well understood. In this subsection make an attempt to quantify the liquid-water effects as these quantities are varied. The benefit of such information is clearly in predicting the performance of different media, namely in the GDL and CCL, whose properties can be classified in relation to contact angle (hydrophobicity/hydrophilicity), permeability and porosity. Let us return to the definitions (21), (22) and (50) and now define the quantities:

$d_{\mathrm{c}}=\frac{\kappa_{\mathrm{c}} \sigma_{\mathrm{c}}}{\mu}, \quad d_{\mathrm{g}}=\frac{\kappa_{\mathrm{g}} \sigma_{\mathrm{g}}}{\mu}$.

Changes in these quantities can arise from changes in several underlying parameters, namely the absolute permeabilities, $\kappa_{\mathrm{c}}$ and $\kappa_{\mathrm{g}}$, the porosities, $\epsilon_{\mathrm{p}}$ and $\epsilon_{\mathrm{g}}$, and the contact angles $\theta_{\mathrm{c}}$ and $\theta_{\mathrm{g}}$. The permeabilities can be decomposed using the KozenyCarman relation, [32],

$\kappa_{\mathrm{c}}=\frac{\epsilon_{\mathrm{p}}^{3} d_{\mathrm{p}, \mathrm{c}}^{2}}{16 K_{\mathrm{c}}\left(1-\epsilon_{\mathrm{p}}\right)^{2}}, \quad \kappa_{\mathrm{g}}=\frac{\epsilon_{\mathrm{g}}^{3} d_{\mathrm{p}, \mathrm{g}}^{2}}{16 K_{\mathrm{g}}\left(1-\epsilon_{\mathrm{g}}\right)^{2}}$,

where $d_{\mathrm{p}, \mathrm{c}}$ and $d_{\mathrm{p}, \mathrm{g}}$ are the mean pore diameters in the CCL and GDL, respectively. $K_{\mathrm{c}}$ and $K_{\mathrm{g}}$ are the so-called Kozeny constants. Ultimately

$d_{\mathrm{g}} \propto d_{\mathrm{p}, \mathrm{g}}, \quad d_{\mathrm{g}} \propto \cos \theta_{\mathrm{c}}^{\mathrm{g}}, \quad d_{\mathrm{g}} \propto \frac{\epsilon_{\mathrm{g}}^{2}}{1-\epsilon_{\mathrm{g}}}$,

so that $d_{\mathrm{g}}$ increases with increasing porosity, contact angle (in the hydrophobic range) and mean pore diameter of the GDL. The same is true for $d_{\mathrm{c}}$ except that a decrease in the contact angle, $\theta_{\mathrm{c}}^{\mathrm{c}}$, leads to an increase in $d_{\mathrm{c}}$. Thus, the results obtained from varying $d_{\mathrm{g}}$ and $d_{\mathrm{c}}$ contain a quite general degree of information. For typical values (those used by Mazumder, [4]), $d_{\mathrm{g}}$ is of the order $10^{-4}-10^{-5}$. In the CCL, where the pores are much smaller $\left(O\left(10^{-7} \mathrm{~m}\right)\right), d_{\mathrm{c}}$ is likely to be one or two orders of magnitude smaller than $d_{\mathrm{g}}$.

Fig. 8 demonstrates the effect on performance and liquidwater accumulation of changes in $d_{\mathrm{g}}$ and $d_{\mathrm{c}}$. In these calculations, both channels are at $a_{\mathrm{w}}=1$ and other parameters, unless otherwise specified, are given in Table 1. It can be seen from the first of these figures that increases in $d_{\mathrm{g}}$ and $d_{\mathrm{c}}$ lead to decreases in the maximum saturation and increases in the minimum saturation (minimum at $x=0$ and maximum at $x=L_{2}$ ), accompanied by increases in the current density, measured at $0.1 \mathrm{~V}$. Changes in $d_{\mathrm{g}}$, at fixed $d_{\mathrm{c}}$, appear to have a more dramatic effect than 

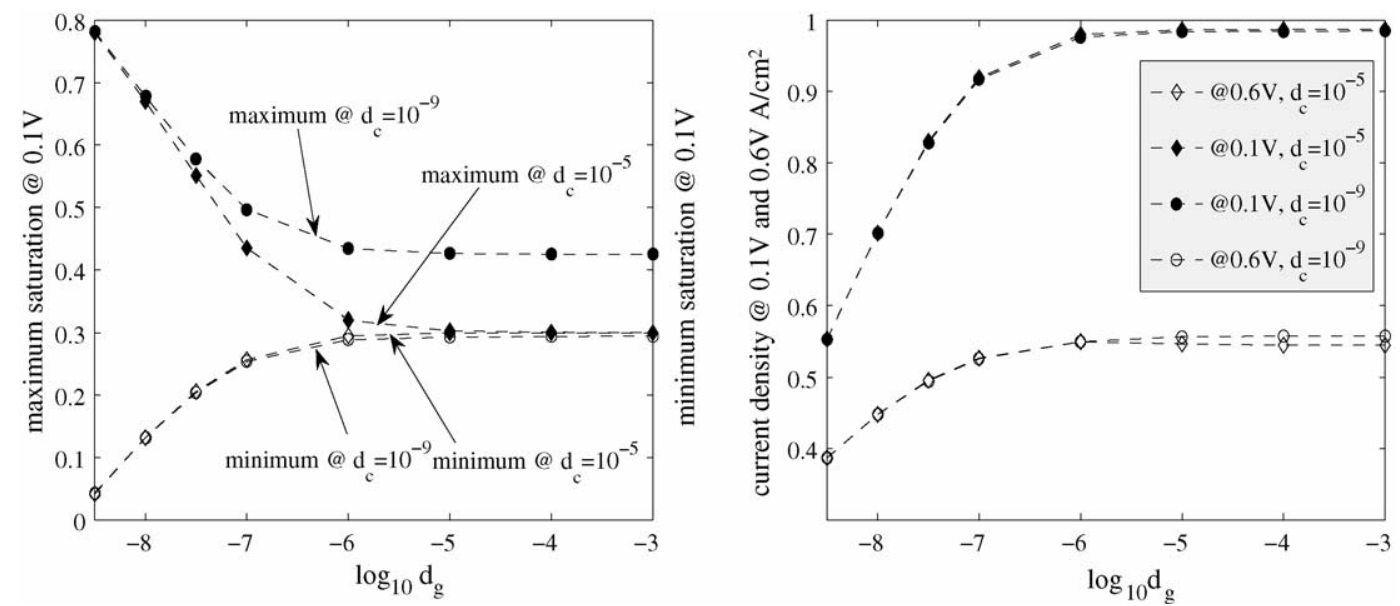

Fig. 8. The effect on the maximum saturation and current density at 0.1 and $0.6 \mathrm{~V}$ of changes in the contact angles, absolute permeabilities and porosities of the CCL and GDL. $d_{\mathrm{g}}$ and $d_{\mathrm{c}}$ are defined in (60) and both channels are at $a_{\mathrm{w}}=1$.

changes in $d_{\mathrm{c}}$, at fixed $d_{\mathrm{g}}$, particularly on the minimum saturation. It can further be seen that for a fixed $d_{\mathrm{c}}$ there is a lower limit to the saturation and an upper limit to the current density as $d_{\mathrm{g}}$ is increased. The difference in the lower limit of maximum saturation as $d_{\mathrm{c}}$ is increased is quite appreciable, but the effect on the current density, measured at both 0.1 and $0.6 \mathrm{~V}$, is negligible. It appears then that changes in the contact angle, pore radius and porosity in the GDL have a far greater impact than changes in the same quantities in the CCL (possibly because of the far greater thickness of the GDL). In fact they can alter the accumulation of liquid water and consequently the performance quite dramatically.

We note finally that in the range $-8.5<\log _{10} d_{\mathrm{g}}<-6$, the current density measured at $0.6 \mathrm{~V}$ does not decrease as rapidly as the current density at $0.1 \mathrm{~V}$. This appears once more to be the consequence of the competition between flooding and hydration of the membrane.

\subsection{Effects of GDL and membrane thickness}

The effects of membrane and GDL thickness changes can be inferred from Fig. 9. For these calculations both channels are at $a_{\mathrm{w}}=1$ and other parameters are given in Table 1, unless otherwise stated. The left-hand figure demonstrates the polarization curves for an increasing range of membrane thickness, with a GDL thickness of $200 \mu \mathrm{m}$. It can be seen from the righthand figure that decreasing $L_{\mathrm{m}}$ improves the performance by increasing the slope of the polarization curve. Quite substantial improvements are possible. The right-hand figure demonstrates that decreasing the GDL thickness improves performance. Interestingly, this is despite a simultaneous increase in the maximum saturation. This implies that improving the oxygen supply to the CCL, by reducing the mass-transport resistance from the GDL, can to some extent mitigate the effects of flooding, another example of the competing effects in the MEA.
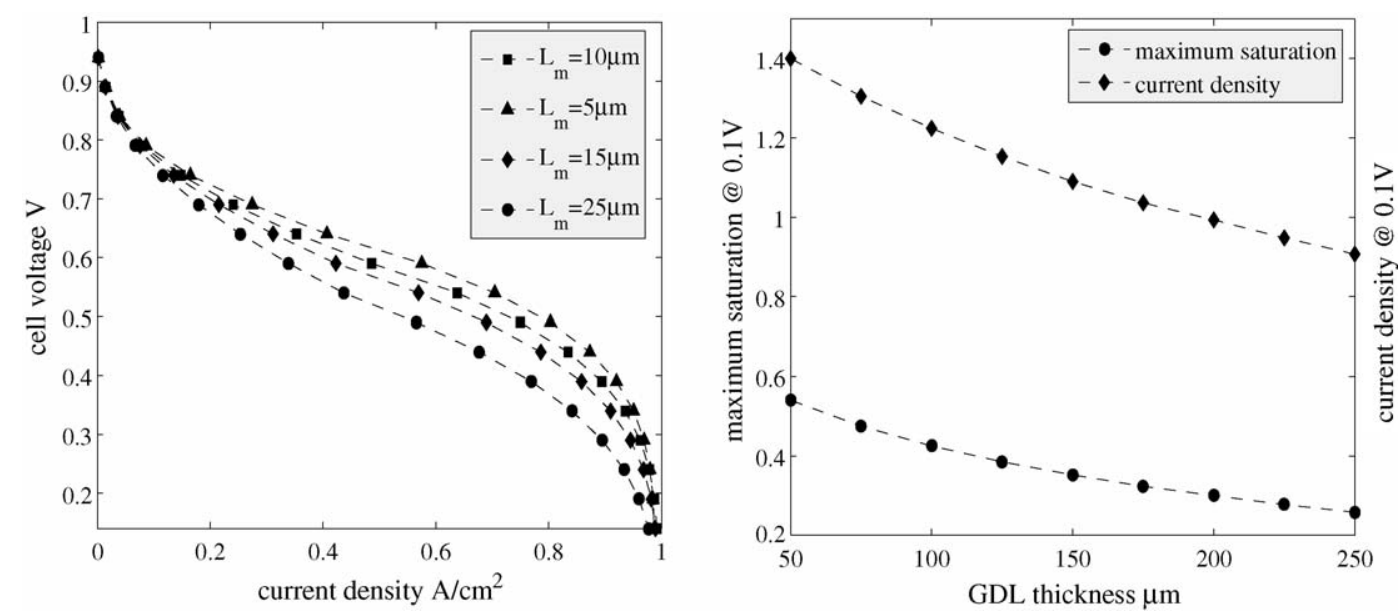

Fig. 9. The left-hand figure shows the polarization curves for a decreasing range of membrane thickness, with a GDL thickness of $200 \mu \mathrm{m}$ and both channels at $a_{\mathrm{w}}=1$. The right-hand figure shows the effect of changes in the GDL thickness on both the maximum saturation and current density measured at a cell voltage of $0.1 \mathrm{~V}$. In this plot $L_{\mathrm{m}}$ is given in Table 1 . 

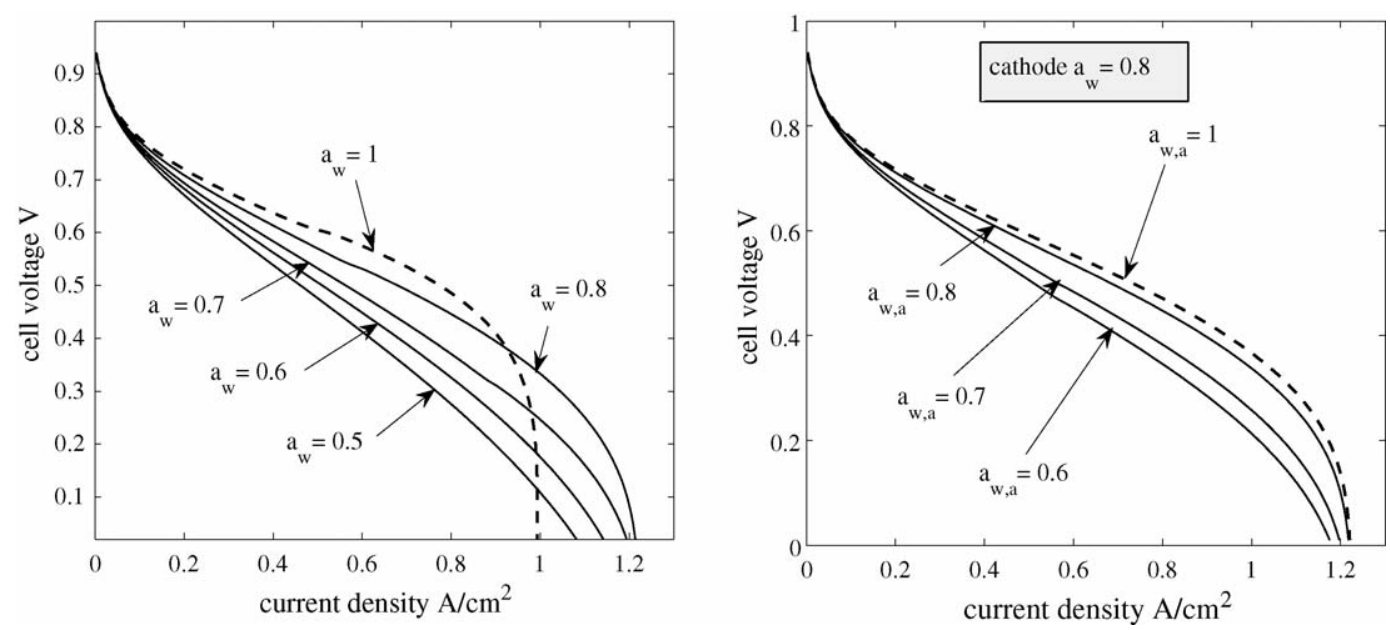

Fig. 10. The effect on the performance of changes in the channel water activity, when maintained at the same value in both channels (left figure) and at different values (right figure).

\subsection{Effects of water activity}

In Fig. 10 we plot the polarization curves for several values of water activity in the channels. The left-hand figure demonstrates that changes in water activity, with the same values in both channels, have a convoluted effect on the performance. At fully humidified conditions the effects of flooding are strongly felt at low cell voltages. However, the performance at high and mid-range voltages is good. At lower humidification levels down to approximately $a_{\mathrm{w}}=0.8$ the performance improves at low cell voltages, with minor deterioration at higher voltages. However, when the water activity is reduced further, the performance deteriorates, particularly at high and mid-range cell voltages but also now at lower cell voltages (compared to $a_{\mathrm{w}}=$ $0.8)$.

Maintaining the cathode-channel water activity and varying that for the anode channel leads to the polarization curves in the right-hand plot of Fig. 10. The best performance is seen at $a_{\mathrm{w}, \mathrm{a}}=1$, with only a minor deterioration down to $a_{\mathrm{w}, \mathrm{a}}=0.8$. Once below this value, the increased resistivity of the membrane degrades the performance substantially, particularly at mid-range cell voltages.

\subsection{Combined effects of temperature and water activity}

Fig. 11 demonstrates the effects of simultaneous changes in the operating temperatures and water activities, at constant pressure in the channels. It appears that at as we increase temperature, changes in water activity are less strongly felt. At $T=60^{\circ} \mathrm{C}$, significant improvements in performance are possible at midrange and high cell voltages if the water activity of the channels is high, as already seen in the previous subsection. At $T=120^{\circ} \mathrm{C}$, while improvements are still possible, they are not as dramatic. One implication of this result is that in some measure it is possible to mitigate the effects of flooding (dramatic deterioration in performance at low cell voltage) by increasing the temperature in the channels, and therefore the average temperature of the system. In Fig. 12 we see the reason for this. At $T=60^{\circ} \mathrm{C}$ the saturation levels are high at low cell voltage, causing the drop in performance seen in Fig. 11. However, due to decreased condensation, at $T=120^{\circ} \mathrm{C}$ even at low cell voltage the saturations levels are too low to lead to flooding.

We note that in Fig. 11, the gas pressure in the channel is constant, so an increase in temperature leads to a decrease in the molar density of the gas, and therefore in total oxygen available. More importantly, an increase in temperature causes a substantial increase in the saturation vapour pressure at high temperatures. At fixed water activity, there are correspondingly large increases in the vapour density. Fig. 13 shows the polarization curves at $a_{\mathrm{w}}=1$ as the temperature is increased from 60 to $120^{\circ} \mathrm{C}$, while the gas pressure, $P_{\mathrm{c}}$, and the concentration of oxygen in the cathode channel, $\bar{C}_{\mathrm{p}}$ are kept constant at $30 \mathrm{psig}(206.842 \mathrm{kPa})$ and $3.75 \mathrm{~mol} \mathrm{~m}^{-3}$, respectively. We see that the current density increases dramatically at low cell voltage because of the reduction in the saturation levels. At higher cell voltages the performance deteriorates, but not substantially, possibly because of the increase in the vapour density and its corresponding effect on the membrane conductivity.

\subsection{Effects of water removal from channel}

To see the effect of the removal of liquid water from the cathode channel we refer to Fig. 14. It is evident that by increasing the removal rate (equivalent to increasing $\Omega$ ) performance improves at $0.1 \mathrm{~V}$. The improvement in performance is accompanied by a decrease in the maximum saturation (over GDL and CCL), and there is an inverse relationship between the flooding level and the current density at this voltage. Moreover, the current density measured at $0.6 \mathrm{~V}$ does not decrease significantly over the same range of $\Omega$. In other words, increasing the removal of liquid water from the channel improves the performance at low cell voltages without significantly affecting the performance at high cell voltages. This point is underlined by Fig. 15. 

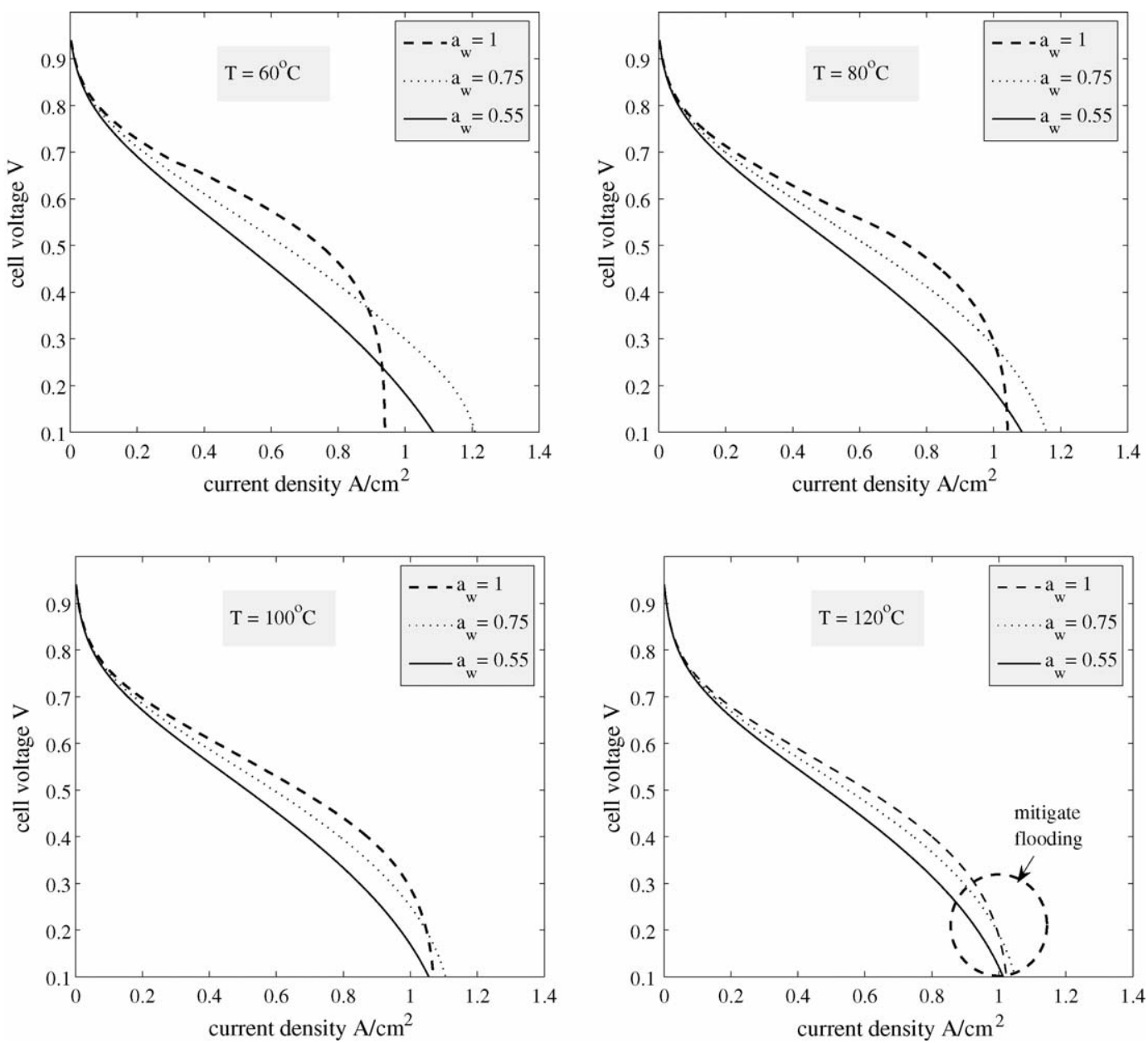

Fig. 11. The effect on the performance of combined changes in the channel water activity and temperature (both channels at the same water activity).

\section{Discussion}

Capturing the effects of (particularly liquid) water is a nontrivial issue in PEMFC modelling. In this paper we have provided the outline of a model that incorporates many of the difficult aspects associated with these effects. In doing so, we found it necessary to explicitly include many of the MEA features that are often subject to approximation, such as a detailed catalyst layer model, temperature variations and the multi-phase nature of the problem.

We have demonstrated through comparison with experiment that the model we have developed qualitatively captures the correct behaviour with respect to flooding and membrane hydration (see Fig. 7). This result, which appears to be absent in previous modelling studies, underlines the competition between hydration of the membrane and flooding, and is clearly an effect which has to be captured in any model that is aimed at predicting or aiding water management. To show the benefits of our modelling approach we performed several studies focussed on water management, particularly with regard to the effects that micro-structure and operating conditions have on performance. We now highlight a few of our findings:
(1) From the results depicted in Fig. 8 and the relationships (60)-(62), it appears that the most influential microstructural property to affect saturation levels is the pore diameter, $d_{\mathrm{p}, \mathrm{g}}$ for the GDL and $d_{\mathrm{p}, \mathrm{c}}$ for the CCL. Provided that they do not approach extreme values $\left(\theta_{\mathrm{c}}^{\mathrm{c}}, \theta_{\mathrm{c}}^{\mathrm{g}} \rightarrow 90^{\circ}\right)$, the contact angles play only a minor role since the maximum saturation is quite insensitive to changes in $d_{\mathrm{c}}$ and $d_{\mathrm{g}}$ that are not at least an order of magnitude. Nevertheless, combined changes in the microstructural properties of the GDL can be employed to improve performance quite substantially.

(2) Reducing the thickness of the membrane improves performance. However, this practice must be treated with caution. If the thickness is reduced by too much, oxygen and hydrogen cross-over will also increase and will lead to severe degradation. Likewise, decreasing the GDL thickness improves performance, with the increased oxygen supply outweighing the increased saturation levels.

(3) From the results of Section 5.5, it is clear that without either changes in the other conditions or external humidification strategies, the resistance of the membrane is prohibitively high if the water activities in the channels are below a critical set of values (around 0.7-0.8). At water activities approach- 

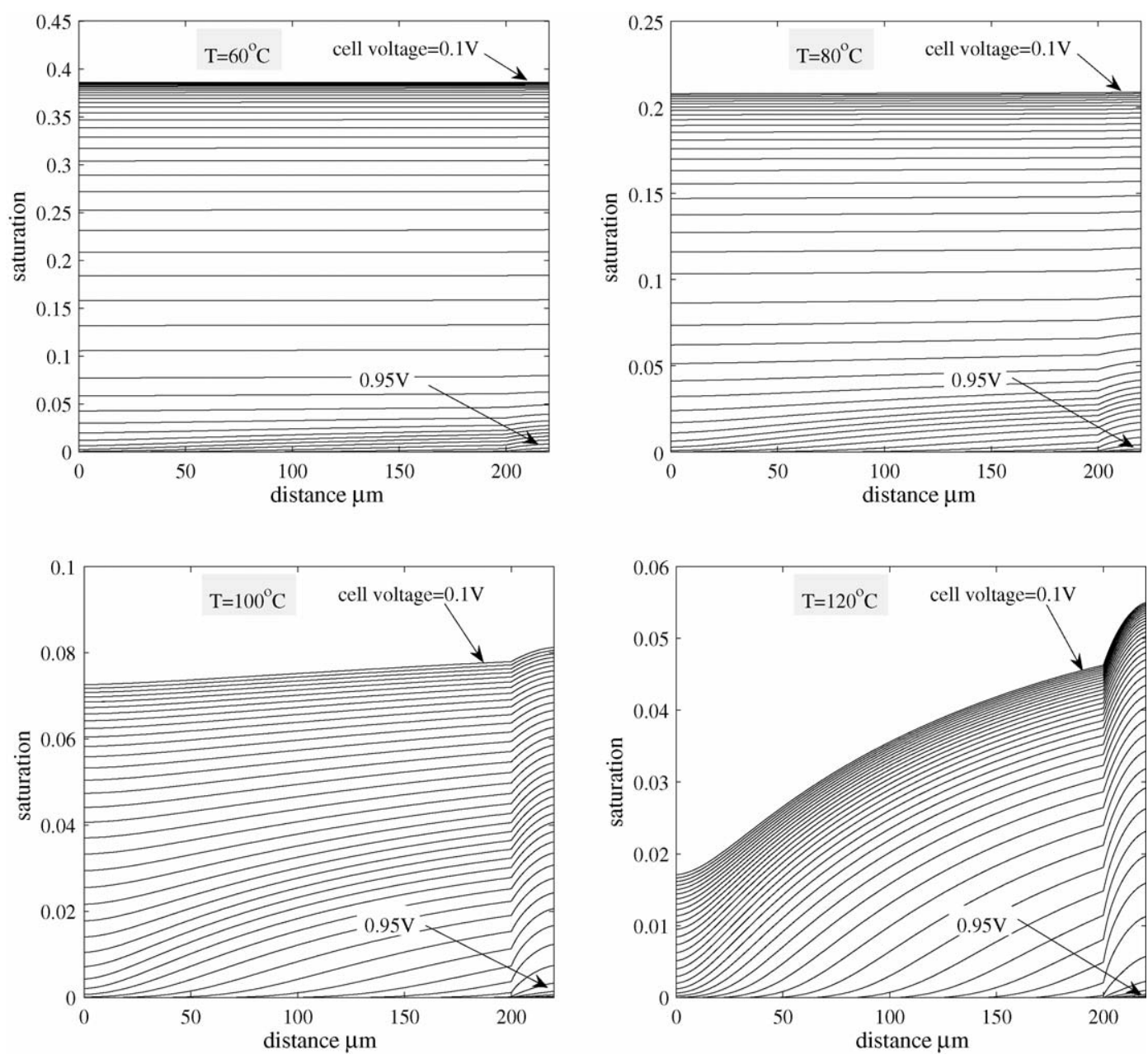

Fig. 12. Saturation profiles corresponding to the calculations at $a_{\mathrm{w}}=1$ in Fig. 11 .

ing unity in both channels, the flooding becomes an issue if the cell is operated at low voltage. However, at mid-range voltages, above approximately $0.4 \mathrm{~V}$, the performance is optimized at $a_{\mathrm{w}}=1$.

(4) If the cell is operated at water activities in the channel that are close to unity, temperature can be used in some measure to offset the effects of flooding by lowering the condensation rate and therefore the saturation levels (see Figs. 11 and 12). From Fig. 13 we see that performance at low cell voltages can therefore be enhanced significantly, while the corresponding deterioration in performance at higher cell voltages is minor. The latter result is possibly due to the increased vapour molar concentration at higher temperature and the corresponding improvement in membrane conductivity.

(5) Fig. 14 suggests that at high water activity significant performance improvements can be made by increasing the removal rate of liquid water from the cathode channel, which although not solely controlled by, is directly linked to the flow rate of gas down the channel. Under these conditions, if no liquid water is removed $(\Omega=0)$ flooding severely impacts the current density at low cell voltage. The greater the removal of the liquid water the higher the current density at

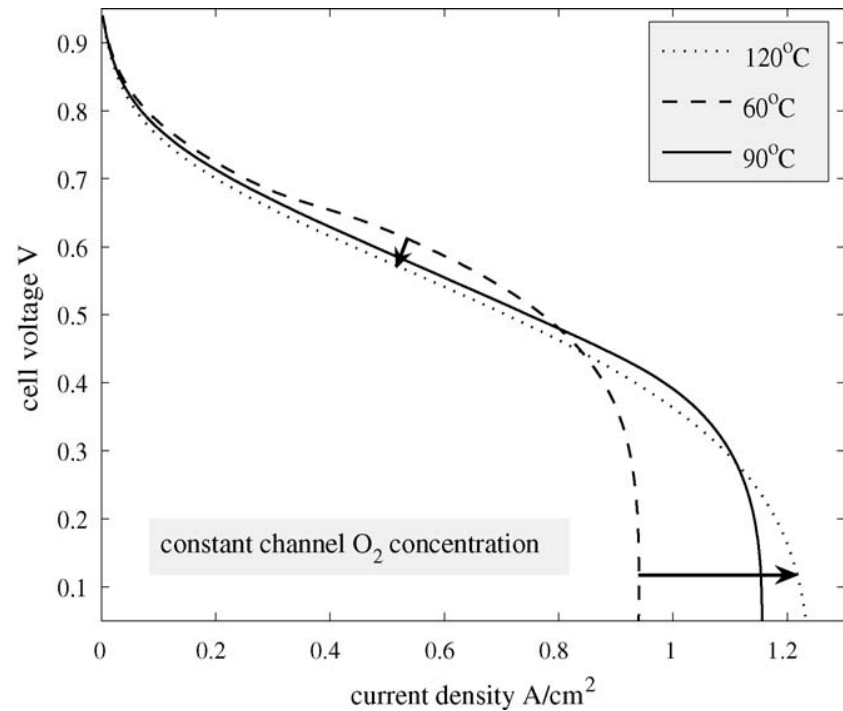

Fig. 13. The effect of temperature increases in the channels (both at the same temperature) with $a_{\mathrm{w}}=1$ in both channels. In these calculations, the oxygen concentration was kept constant as temperature was varied. 


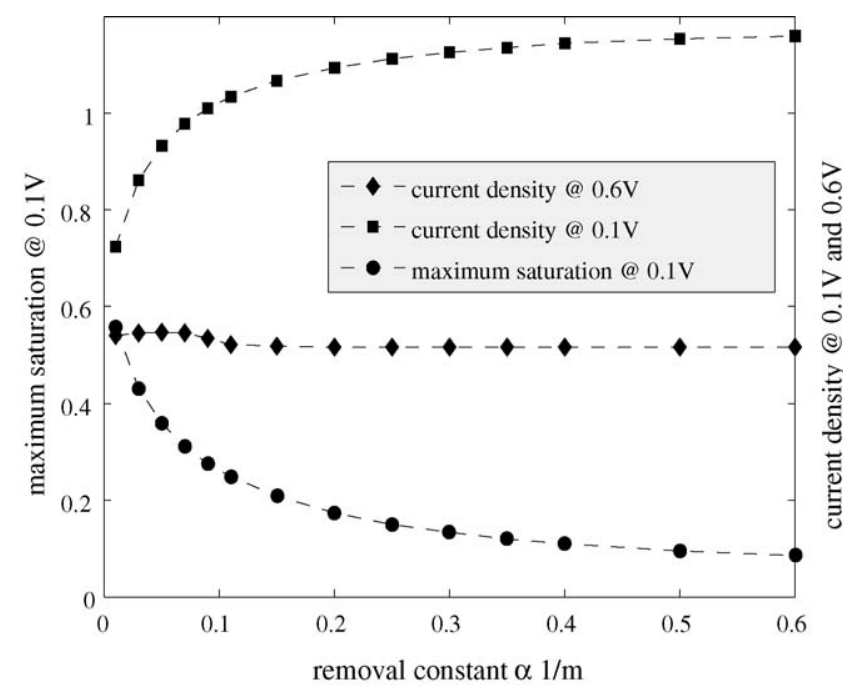

Fig. 14. The effect of the removal constant $\Omega$, defined in (59), on the maximum saturation and the current density measured at 0.1 and $0.6 \mathrm{~V}$. Both channels are at $a_{\mathrm{W}}=1$.

low cell voltage, which eventually plateaus as $\Omega$ increases. Furthermore, the performance at higher cell voltage is not dramatically affected as the removal increases (see Fig. 15).

Though we have concentrated on water effects in this paper there are several other aspects to the model that can be exploited. For example, a parametric study with respect to the microstructural properties of the CCL and conditions under which proton migration is limiting. Our purpose has been to demonstrate the ability of the model to capture complex phenomena. There are several extensions to the model that we are currently pursuing. In order to study effects such as hydrogen and oxygen (as well as water) crossover, the anode catalyst and gas-diffusion layers must be included. In addition, extension to two dimensions will provide details of the non-uniformities down the channels.

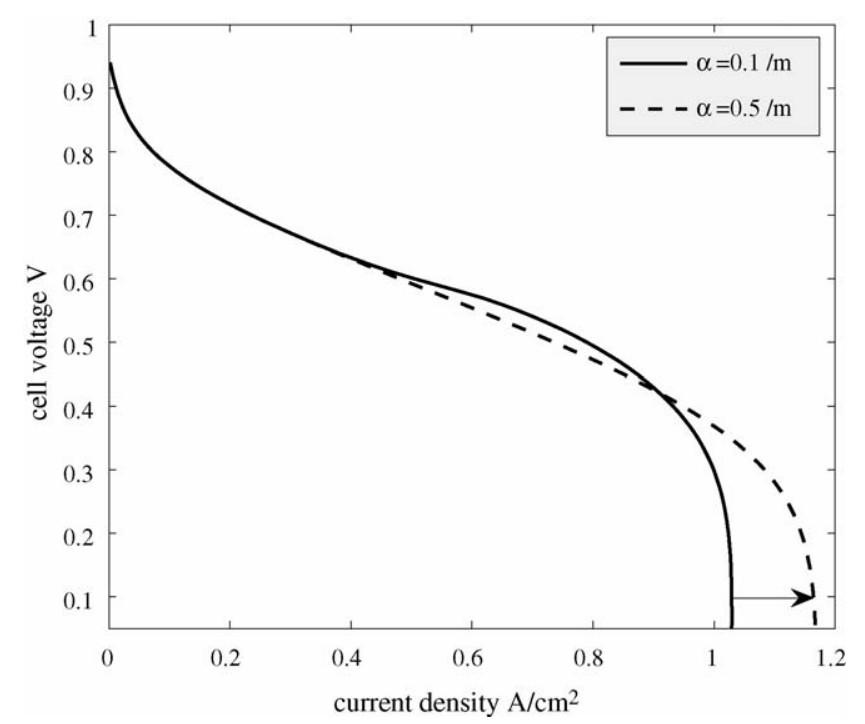

Fig. 15. The effect on the polarization curve of changes in $\Omega$, the removal rate constant.

\section{Acknowledgements}

The authors are grateful to MITACS and Ballard Power Systems for their financial support and experimental facilities. We would particularly like to acknowledge Dr. Jean St. Pierre (formerly of Ballard Power Systems), Professor Brian Wetton and Dr. John Stockie (MITACS) for their involvement in the many fruitful discussions leading to this paper.

\section{References}

[1] J. Cao, N. Djilali, Computational simulation of water transport in PEM fuel cells using an improved membrane model, Proceedings of the 10th Canadian Hydrogen Conference (2000) 447-456.

[2] W. He, J. Yi, T.V. Nguyen, Two-phase flow model of the cathode of PEM fuel cells using interdigitated flow fields, AIChE J. 46 (10) (2000) 20532064.

[3] G. Lin, W. He, T. Van Nguyen, Modelling liquid water effects in the gas diffusion and catalyst layers of the cathode of a PEM fuel cell, J. Electrochem. Soc. 151 (11) (2004) A1999-A2006.

[4] S. Mazumder, J.V. Cole, Rigorous 3D mathematical modelling of PEM fuel cells: 2 model predictions with liquid water transport, J. Electrochem. Soc. 150 (11) (2004) A1510-A1517.

[5] D. Natarajan, T.V. Nguyen, A two-dimensional, two-phase, multicomponent, transient model for the cathode of a proton exchange membrane fuel cell using conventional gas distributors, J. Electrochem. Soc. 148 (12) (2001) A1324-A1335.

[6] N.P. Siegel, M.W. Ellis, D.J. Nelson, M.R. von Spakovsky, Single domain PEMFC model based on agglomerate catalyst geometry, J. Power Sources 115 (1) (2003) 81-89.

[7] N.P. Siegel, M.W. Ellis, D.J. Nelson, M.R. von Spakovsky, A twodimensional computational model of a PEMFC with liquid water transport, J. Power Sources 128 (1) (2004) 173-184.

[8] L.B. Wang, N.I. Wakayama, T. Okada, Numerical simulation of enhancement of mass transfer in the cathode electrode of a PEM fuel cell by magnet particles deposited in the cathode-side catalyst layer, Chem. Eng. Sci. 60 (2005) 4453-4467.

[9] Z.H. Wang, C-Y. Wang, K.S. Chen, Two-phase flow and transport in the air cathode of proton exchange membrane fuel cells, J. Power Sources 94 (2001) 40-50.

[10] S. Lister, G. McLean, Review: PEM fuel cell electrodes, J. Power Sources 130 (2004) 61-76.

[11] F. Liu, B. Yi, D. Xing, J. Yu, Z. Hou, Y. Fu, Development of novel selfhumidifying composite membranes for fuel cells, J. Power Sources 124 (2003) 81-89.

[12] M. Uchida, Y. Aoyama, N. Eda, O. Akira, Investigation of the microstructure in the catalyst layer and effects of both perfluorosulfonate ionomer and PTFE-loaded carbon on the catalyst layer of polymer electrolyte fuel cells, J. Electrochem. Soc. 142 (12) (1995) A4143-A4149.

[13] M. Uchida, Y. Fukuota, Y. Sugawara, H. Ohara, O. Akira, Improved preparation process of very-low-platinum-loading electrodes for polymer electrolyte fuel cells, J. Electrochem. Soc. 145 (11) (1998) A3708A3713.

[14] A.Z. Weber, J. Newman, Modelling transport in polymer-electrolyte fuel cells, Chem. Rev. 104 (2004) 4679-4726.

[15] K. Broka, P. Ekdunge, Modelling the PEM fuel cell cathode, J. Appl. Electrochem. 27 (1997) 281-289.

[16] Q. Guo, R. White, A steady-state impedance model for a PEMFC cathode, J. Electrochem. Soc. 151 (4) (2004) E133-E149.

[17] F. Jaouen, G. Lindbergh, G. Sundholm, Investigation of mass-transport limitations in the solid polymer fuel cell cathode: I. mathematical model, J. Electrochem. Soc. 149 (4) (2002) A437-A447.

[18] S. Mazumder, J.V. Cole, Rigorous 3D mathematical modelling of PEM fuel cells: 1 model predictions without liquid water transport, J. Electrochem. Soc. 150 (11) (2004) A1503-A1509. 
[19] W. Sun, B.A. Peppley, K. Karan, An improved two-dimensional agglomerate cathode model to study the influence of catalyst layer structural parameters, Electrochim. Acta 50 (2005) 3359-3374.

[20] K. Yin, Parametric study of proton-exchange membrane fuel-cell cathode using an agglomerate model, J. Electrochem. Soc. 152 (3) (2005) A583A593.

[21] R. Aris, The Mathematical Theory of Diffusion and Reaction in Permeable Catalysts, vol. 1. The Theory of the Steady State, Oxford University Press, 1975.

[22] M. Eikerling, A.A. Kornyshev, Modelling the performance of the cathode catalyst layer of polymer electrolyte fuel cells, J. Electroanal. Chem. 453 (1998) 89-106.

[23] M. Eikerling, A.A. Kornyshev, Electrochemical impedance of the cathode catalyst layer in polymer electrolyte fuel cells, Electroanal. Chem. 475 (1999) 107-123.

[24] D.B. Genevey, Transient model of heat, mass and charge transfer as well as electrochemistry in the cathode catalyst layer of a PEMFC, Master's Thesis, Virginia Polytechnic Institute, 2004.

[25] H.-K. Hsuen, Mechanistic approach to performance equations for cathodes in polymer electrolyte fuel cells, J. Power Sources 123 (2003) 2636.

[26] D. Song, Q. Wang, Z. Liu, T. Navessin, M. Eikerling, S. Holdcroft, Numerical optimization study of the catalyst layer of a PEM fuel cell cathode, J. Power Sources 126 (2004) 104-111.

[27] C.Y. Sukkee Um, K.S. Wang, Chen, Computational fluid dynamics modeling of proton exchange membrane fuel cells, J. Electrochem. Soc. 147 (12) (2000) A4485-A4493.

[28] Q. Wang, M. Eikerling, D. Song, Z. Liu, Structure and performance of different types of agglomerates in cathode catalyst layer of PEM fuel cells, J. Electroanal. Chem. 573 (2004) 61-79.

[29] H. Ju, H. Meng, C.-Y. Wang, A single-phase, non-isothermal model for PEM fuel cells, Int. J. Heat Mass Transfer 148 (7) (2005) 1303 $-1315$.

[30] H. Weng, C.-Y. Wang, Model of two-phase flow and flooding dynamics in polymer electrolyte fuel cells, J. Electrochem. Soc. 152 (9) (2005) A1733A1741.

[31] C.-Y. Wang, P. Cheng, Multiphase flow and heat transfer in porous media, J. Power Sources 30 (1997) 93-196.

[32] J-H. Nam, M. Kaviany, Effective diffusivity and water-saturation distribution in single- and two-layer PEMFC diffusion medium, Int. J. Heat Mass Trans. 46 (2003) 4595-4611.

[33] U. Pasaogullari, C-Y. Wang, K.S. Chen, Two-phase transport in polymer electrolyte fuel cells with bilayer cathode diffusion media, J. Electrochem. Soc. 152 (8) (2005) A1574-A1582.

[34] E. Middelman, Improved PEM fuel cell electrodes by controlled selfassembly, Fuel Cells Bull. (2002) 9-12.

[35] H. Mizuhata, N. Shin-ichi, T. Yamaguchi, Morphological control of PEMFC electrode by graft polymerization of polymer electrolyte onto platinum-supported carbon black, J. Power Sources 138 (2004) 25-30.

[36] M. Mathias, J. Roth, J. Fleming, W. Lehnert, Diffusion media materials and characterization, in: W. Vielstich, A. Lamm, H. Gasteiger (Eds.), Handbook of Fuel Cells-Fundamentals, Technology and Applications, vol. 3, John Wiley \& Sons, 2003 (Chapter 46).

[37] B. Bird, W. Stewart, E. Lightfoot, Transport Phenomena, John Wiley and Sons, 2002.

[38] Z. Ogumi, Z. Takehara, S. Yoshizawa, Gas permeation in spe method I. oxygen permeation through nafion and neosepta, J. Electrochem. Soc. 131 (4) (1984) A769-A772.

[39] T.E. Springer, T.A. Zawodinski, S. Gottesfeld, Polymer electrolyte fuel cell model, J. Elecrochem. Soc. 138 (4) (1991) A2334-A2341.

[40] T. Thapman, S. Malhotra, H. Tang, R. Datta, Modeling of conductive transport in proton-exchange membranes for fuel cells, J. Elecrochem. Soc. 147 (9) (2000) A3242-A3250.

[41] P. Berg, K. Promislow, J. St. Pierre, J. Stumper, B. Wetton, Water management in PEM fuel cells, J. Electrochem. Soc. 151 (3) (2004) A341-A353.

[42] S. Motupally, A.J. Becker, J.W. Weidner, Diffusion of water in nafion 115 membranes, J. Electrochem. Soc. 147 (9) (2000) A3171-A3177.

[43] J.T. Hinatsu, M. Mizuhata, H. Takenaka, Water uptake of perfluorosulfonic acid membranes from liquid water and water vapor, J. Elecrochem. Soc. 141 (1994) A1493-A1497.

[44] S. Ge, X. Li, B. Yi, I.M. Hsing, Absorption, desorption and transport of water in polymer electrolyte membranes for fuel cells, J. Electrochem. Soc. 152 (6) (2005) A1149-A1157.

[45] A.Z. Weber, J. Newman, Transport in polymer-electrolyte membranes II. mathematical model, J. Elecrochem. Soc. 151 (2) (2004) A311-A325.

[46] S. Mazumder, A generalized phenomenological model and database for the transport of water and current in polymer electrolyte membranes, J. Electrochem. Soc. 152 (8) (2005) A1633-A1644.

[47] H. Weng, C-Y. Wang, Electron transport in pefcs, J. Electrochem. Soc. 151 (3) (2004) A358-A367.

[48] X.G. Yang, F.Y. Zhang, A.L. Lubawy, C.Y. Wang, Visualization of liquid water transport in a PEFC, Electrochem. Solid-State Lett. 7 (11) (2004) A408-A411.

[49] M.V. Williams, H.R. Kunz, J.M. Fenton, Influence of convection through gas-diffusion layers on limiting current in PEM fcs using a serpentine flow field, J. Elecrochem. Soc. 151 (10) (2004) A1617-A1627.

[50] T. Van Nguyen, W. He, Interdigitated flow field design, in: W. Vielstich, A. Lamm, H. Gasteiger (Eds.), Handbook of Fuel Cells-Fundamentals, Technology and Applications, vol. 3, John Wiley \& Sons, 2003 (Chapter 46).

[51] R.H. Perry, D.W. Green, J.O. Maloney, Perry's Chemical Engineers Handbook, McGraw-Hill, 1984.

[52] Suk Won Cha, Scaling effects of flow channels in fuel cells, PhD Thesis, Stanford University, 2003.

[53] M.J. Lampinen, M. Fomino, Analysis of free energy and entropy changes for half-cell reactions, J. Electrochem. Soc. 140 (12) (1993) A3537 $-\mathrm{A} 3546$.

[54] Q. Guo, V.A. Sethuraman, R. White, Parameter estimates for a PEMFC cathode, J. Electrochem. Soc. 151 (7) (2004) A983-A993.

[55] B. Carnes, N. Djilal, Systematic parameter estimation for PEM fuel cell models, J. Power Sources 144 (1) (2005) 83-93. 